\title{
ASSET PRICING WITH NO EXOGENOUS PROBABILITY MEASURE
}

\author{
GIANLUCA CASSESE
}

\begin{abstract}
In this paper we propose a model of financial markets in which agents have limited ability to trade and no probability is given from the outset. In the absence of arbitrage opportunities, assets are priced according to a probability measure that lacks countable additivity. Despite finite additivity, we obtain an explicit representation of the expected value with respect to the pricing measure, based on some new results on finitely additive measures. From this representation we derive an exact decomposition of the rsik premiu as the sum of the correlation of returns with the market price of risk and an additional term, the purely finitely additive premium, related to the jumps of the return process. We also discuss the implications of the absence of free lunches.
\end{abstract}

\section{Introduction.}

Continuous time financial models adopt a wide definition of the trading activity of agents, no matter the degree of market imperfections considered. The ground for such definition is laid by two basic assumptions (henceforth jointly referred to as the traditional setting): that a probability measure is given and that, with reference to this, gains from trade may be modeled as semimartingales. This framework has fostered a large number of important results in asset pricing and portfolio theory and represents the backbone of modern financial theory. In all such developments it is therefore implicit the view of investors as agents of considerably refined ability, both in the assessment of uncertainty and in the trading of assets. The delta hedging strategy of Black and Scholes [8], a standard textbook case regardless of its overwhelming operational complexity, is a good case in point.

We present in this paper a theory of financial prices in continuous time and with a general state space with two distinctive features: no probability measure is given (as in the original spirit of Arrow's [4] model) and the trading of assets is considerably restricted. More precisely, we will only consider trading strategies which $(i)$ extend over a finite time horizon, $(i i)$ prescribe rebalancing positions a finite number of times and (iii) are contingent on a finite number of possible scenarios. On the other hand, given our focus on the constraints to financial activity coming from the subjective side, we assume that markets are free of imperfections and that investment (discounted) returns are bounded. In sections 3 to 6 we analyze the implications ensuing from the basic economic principle of absence of arbitrage opportunities. We obtain versions of the Fundamental Theorem of Asset Pricing and of the Capital Asset Pricing Model that, while comparing with the corresponding results in the traditional setting, exhibit at the same time differences of significant economic content.

The assumption of a given probability measure rests on the possibility that this may be retrieved either from historical data or from individual preferences over uncertain outcomes. Neither argument is however free of criticisms. On the one side whenever time series are not sufficiently stationary averaging data may

Date: July 26, 2006.

Key words and phrases. Arbitrage, Asset pricing, CAPM, Finitely additive measures, Finitely additive conditional expectation, Free lunch, Fundamental theorem of asset pricing, Martingale measure, Semimartingales. 
lead to unstable estimates or to severe undervaluation of rarely occurring events. This is particularly relevant as non stationarity of financial time series is one of the of stylized facts in financial analysis and it contributes significantly to explaining puzzles, such as the equity premium, see e.g. [13]. On the other hand, a vast stream of literature, taking moves from paradoxes of expected utility, has cast doubt on the idea that a probabilistic assessment of uncertainty be implicit in preferences. Although in experimental psychology, subadditivity is a long-standing evidence (see [41] and [42] for pioneering contributions), more recent theoretical work has laid ground for models in which choice is not based on probability but rather on set functions with considerably poorer structure. Examples are Choquet expected utility [38], case-based decision making [22] and support theory [43].

The absence of arbitrage opportunities delivers a number of conclusions. First, there exists a pricing measure, $m$, (a risk neutral measure, in the traditional terminology) that will in general only be finitely additive. Second, associated to $m$ is a countably additive probability measure $P$, termed the representing measure. The role of this compares to that of the "physical" or "objective" measure in traditional models, although in our approach $P$ is endogenous and it is generated by the pricing measure, rather than the other way round. In some sense one should think of the representing measure as the measure the investor would adopt were he to write a mathematical model of financial markets. In fact $P$ permits an explicit and analytically tractable representation of the pricing rule, Proposition 2, and asset returns turn out being $P$ semimartingales, Theorem 7 . These findings allow to overcome some of the difficulties involved in finitely additive expectation and, in some sense, restore the traditional properties of financial models but in purely endogenous terms. Much of these developments are based on some new results on finitely additive probabilities obtained in [9], a measure decomposition for filtered probability spaces, Lemma 2, and a notion of conditional expectation for finitely additive probabilities, Proposition 1.

Our model also contains strong financial implications. In particular we show that risk premia decompose exactly into the sum of two terms, the correlation of returns with a market density process and a component arising from the lack of countable additivity of the pricing measure and thus referred to as the purely finitely additive premium. This decomposition implies that traditional, $C A P M$-like explanations of risk premia which are based solely on the market price of risk need not be correct. We show that the purely finitely additive premium only depends on the discontinuous part of the return process although it need not be itself a pure jump process. This suggests that unpredictable discontinuities, such as market crashes, may have a long lasting influence on equity premia. Many a paper has considered extending the $C A P M$ to the case of discontinuous asset returns (see [5], [28] and [39], among others) but in the traditional setting there cannot be but one risk factor unless ad hoc structures of individual preferences are invoked, e.g. [17] and [19]. On the other hand, it has long been recognized that the existence of more than one factor could be responsible for the poor performance of the $C A P M$ in empirical terms. Our findings contribute thus to the debate on risk premia by illustrating the role of finite additivity.

The present paper is organized as follows. After describing the model, in section 2, we prove in section 3 the existence of the pricing measure $m$ and discuss its properties. In section 4 we derive the existence of a representing measure $P$ associated to $m$, introduce the concept of finitely additive conditional expectation and derive an explicit characterization of the pricing rule. This result allows to establish, in section 5 , that asset returns are $P$ semimartingales and to obtain the risk premia decomposition discussed above. In section 6 we investigate the absence of free lunches condition, introduced in [16]. 


\section{The Model.}

2.1. Set-up and Notation. The state space is described by an arbitrary set $\Omega$. For each date $t \in \mathbb{R}_{+}, \mathcal{F}_{t}$ is a $\sigma$ algebra of subsets of $\Omega$ representing the information available at time $t$. We posit that $\left(\mathcal{F}_{t}: t \in \mathbb{R}_{+}\right)$ is a right continuous filtration and that $\mathcal{F}_{0}=\sigma \mathcal{N}$, the $\sigma$ algebra generated by the collection $\mathcal{N}$, to be introduced below. We denote $\mathcal{F}=\sigma \bigcup_{t \in \mathbb{R}_{+}} \mathcal{F}_{t}$ and $\mathcal{T}$ is the set of stopping times of the filtration. If $X=\left(X_{t}: t \in \mathbb{R}_{+}\right)$and $\tau \in \mathcal{T}$, by $X^{\tau}$ we indicate the "stopped" process $\left(X_{t \wedge \tau}: t \in \mathbb{R}_{+}\right)$. A process $X$ is càdlàg if the deterministic function $t \rightarrow X(\omega, t)$ is right continuous with left limits for all $\omega \in \Omega$. $\tilde{\mathcal{F}}$ is the product $\sigma$ algebra $\sigma \mathcal{F} \otimes \mathcal{B}\left(\mathbb{R}_{+}\right)$on $\Omega \times \mathbb{R}_{+}$- where $\mathcal{B}\left(\mathbb{R}_{+}\right)$is the Borel $\sigma$ algebra over $\mathbb{R}_{+}-$and $\mathcal{P}$ is the predictable $\sigma$ algebra of subsets of $\Omega \times \mathbb{R}_{+}$(for standard terminology on stochastic processes we refer to [27] or [34]). The stochastic integral of $\theta$ with respect to $X$, whenever well defined, is indicated at will by $\int \theta d X$ or $\theta$.X. We do not distinguish between a set and its indicator (so that by $F G$ we may denote the sets $F \cap G$ or $F \times G$ as well as their indicators); if $\mathcal{G}$ is a collection of subsets of $\Omega$, by $\mathfrak{L}(\mathcal{G})$ we indicate the linear space spanned by the indicators of sets in $\mathcal{G} \cdot \mathfrak{B}(\mathbb{H}, \mathcal{H})$ denotes the space of all bounded, $\mathcal{H}$ measurable, real valued functions on some set $\mathbb{H}$ as in $[20$, p. 257] although we will more often adopt the notation $\mathfrak{B}(\mathcal{H})$, when reference to $\mathbb{H}$ is clear. By $b a(\mathcal{F})$ and $c a(\mathcal{F})$ we mean, as usual, the spaces of additive and countably additive set functions on $\mathcal{F}$ of bounded variation; by $\mathbb{P}(\mathcal{F})$ and $\mathbb{P}_{b a}(\mathcal{F})$ we indicate the set of probability measures and of finitely additive probability measures on $\mathcal{F}$ respectively. We also adopt the lattice notation $f^{+}=f \vee 0$ and $f^{-}=-f \vee 0$.

2.2. Negligible Events. Some recent alternatives to expected utility, referred to in the introduction, suggest that preferences need not embody a truly probabilistic assessment. Moreover, substantial experimental evidence (see [14] and [15] for comprehensive reviews) documents the deep influence on individual choice of psychological elements such as the framing of decisions which often lead to attach importance to events in a selective way - and may thus result in market phenomena such as over- or under-reaction. Although preferences are not the focus of this work, we make a step towards more general models of choice introducing the notion of negligibility. This is defined with reference to a collection $\mathcal{N}$ of subsets of $\Omega$, the class of negligible events, which is given a priori and that, interpreted from the point of view of a decision maker, should be viewed as a description of those events that do not affect his choice. Several examples come to mind. The most familiar one is the class $\mathcal{N}_{Q}$ of null sets generated by some given $Q \in \mathbb{P}(\mathcal{F})$, as in the traditional setting in financial theory or in von Neumann and Morgestern model of expected utility. Alternative approaches to choice under uncertainty may as well be reconciled with this concept. Among these, the notion of qualitative probability introduced by Savage [37], in terms of which $\mathcal{N}$ would consist of all events deemed as likely as $\varnothing$ by the decision maker, and Choquet expected utility in which the attitude towards uncertainty is associated with a subadditive capacity and $\mathcal{N}$ may thus amount to the collection of sets which are null with respect to the capacity. We may however also consider phenomena of incompleteness of preferences, such as situations in which agents are simply unable to carry out a proper assessment of the likelihood of events. The source of negligibility may in other words lie in some form of bounded rationality. $\mathcal{N}=\varnothing$ is yet another possibility. ${ }^{1}$

We retain from these examples three basic properties, listed in the following

Assumption 1. The collection $\mathcal{N}$ satisfies the following properties:

\footnotetext{
${ }^{1}$ After this paper was ultimated, I came across the work of Bättig and Jarrow [7] in which a collection of sets similarly defined (the null sets in their terminology) is introduced. In their results, however, the authors seem to be interested only in the special case in which $\mathcal{N}$ is generated by a probability measure.
} 
(i) $\Omega \notin \mathcal{N}$;

(ii) $A \in \mathcal{N}$ and $B \subset A$ imply $B \in \mathcal{N}$;

(iii) $A, B \in \mathcal{N}$ implies $A \cup B \in \mathcal{N}^{2}$.

While property $(i)$ avoids trivial cases, the brief discussion above supports property (ii). As for (iii), although $\mathcal{N}$ need not be closed with respect to countable unions (as is the case in [7]), it is essential for what follows that it is so for finite unions, as will soon be clear.

Definition 1. Let $f, g \in \mathbb{R}^{\Omega}$. Then $f \geq g$ up to negligibility (shorted as u.n.) whenever $\{f-g \leq-\eta\} \in \mathcal{N}$ for each $\eta>0^{3}$.

Likewise, by $f=g$ u.n. we mean $f \geq g$ u.n. and $g \geq f$ u.n. while $f>g$ u.n. whenever $f \geq g$ u.n. but not $g \geq f$ u.n.. The binary relation $\geq$ u.n. extends the notion of negligibility from sets to random quantities:

Definition 2. Let $f \in \mathbb{R}^{\Omega}$. Then $f$ is negligible whenever $f=0$ u.n.

Remark 1. Given our choice to identify sets with indicators, it is important to note that for $F \subset \Omega, F \geq 0$ u.n. - as $\{F \leq-\eta\}=\varnothing$ - and that $F=0$ u.n. if and only if $F \in \mathcal{N}-$ as $\{F \geq \eta\}=F$ for all $1 \geq \eta>0$. Moreover, it is straightforward from Definition 1 that $\geq$ u.n. is reflexive and translation invariant - i.e. $f \geq g$ u.n. is equivalent to $f+h \geq g+h$ u.n. for all $f, g, h \in \mathbb{R}^{\Omega}$.

More properties are established in the following (by $\overline{\mathfrak{L}(\mathcal{N})}$ we denote the closure of $\mathfrak{L}(\mathcal{N})$ in the norm topology of $\mathfrak{B}(\mathcal{F})$ ).

Lemma 1. Assumption 1 is a necessary and sufficient condition for $\geq$ u.n. to satisfy the following properties:

(a) there exists $f \in \mathbb{R}^{\Omega}$ such that $f>0$ u.n.;

(b) $f \geq 0$ implies $f \geq 0$ u.n.;

(c) $\geq$ u.n. is transitive (and therefore a preorder).

Moreover, $f \geq 0$ u.n. if and only if $r \wedge f^{-} \in \overline{\mathfrak{L}(\mathcal{N})}$ for each $r>0$.

Proof. Consider properties (i)-(iii) listed in Assumption 1. (a) follows from $(i)$ - see remark 1; $(b)$ is a consequence of $\varnothing \in \mathcal{N}$ implicit in (ii). Let $f, g \in \mathbb{R}^{\Omega}$ and $\eta>0$. The inclusion $\{f+g \leq-\eta\} \subset$ $\{f \leq-\eta / 2\} \cup\{g \leq-\eta / 2\}$ together with (iii) implies that $f+g \geq 0$ u.n. whenever $f \geq 0$ u.n. and $g \geq 0$ u.n.. (c) then follows from translation invariance.

Assume now $(a)-(c)$. If $f>0$ u.n. then $\{f \geq \eta\} \notin \mathcal{N}$ for some $\eta>0$ so that $0<\{f \geq \eta\}$ u.n. while $\{f \geq \eta\} \leq \Omega$ follows from $(b)$ : by $(c)$, then, $\Omega>0$ u.n. i.e. $\Omega \notin \mathcal{N}$. If $G \subset F$ and $F \in \mathcal{N}$ then by $(b),(c)$ and remark $10 \leq G \leq F \leq 0$ u.n. so that $G \in \mathcal{N}$ i.e. (ii) holds. If $F, G \in \mathcal{N}$ then $G \leq 0$ u.n., $F \leq 0$ u.n. and, by translation invariance, $F+G \leq F$ u.n.: (c) then implies $F+G \leq 0$ u.n. i.e. property (iii).

If for each $r, \eta>0$ there is $g_{r, \eta} \in \mathfrak{L}(\mathcal{N})$ such that $\left|\left(r \wedge f^{-}\right)-g_{r, \eta}\right|<\frac{1}{2}(r \wedge \eta)$ then

$$
\{f \leq-\eta\}=\left\{f^{-} \geq \eta\right\} \subset\left\{r \wedge f^{-} \geq r \wedge \eta\right\} \subset\left\{g_{r, \eta} \geq \frac{1}{2}(r \wedge \eta)\right\} \in \mathcal{N}
$$

\footnotetext{
${ }^{2}$ In set theoretic terminology $\mathcal{N}$ is therefore an ideal.

${ }^{3}$ If (iii) in Assumption 1 were replaced by the property that $\bigcup_{n} A_{n} \in \mathcal{N}$ whenever $A_{n} \in \mathcal{N}$ for all $n \in \mathbb{N}$ then $f \geq g$ u.n. would simply amount to $\{f<g\} \in \mathcal{N}$.
} 
Conversely, if $f \geq 0$ u.n. then $\left\{f^{-} \geq \eta\right\} \in \mathcal{N}$ so that

$$
g_{n}=\sum_{i=1}^{2^{n}} i r 2^{-n}\left\{i r 2^{-n} \leq\left(r \wedge f^{-}\right)<(i+1) r 2^{-n}\right\} \in \mathfrak{L}(\mathcal{N})
$$

and $\left|\left(r \wedge f^{-}\right)-g_{n}\right| \leq 2^{-n}$.

This Lemma allows to interpret Assumption 1 by the properties it induces on the binary relationship $\geq$ u.n.. In particular, as clearly emerges from the proof, $\geq$ u.n. is a preorder - and consequently $=$ u.n. an equivalence relation - if and only if $\mathcal{N}$ is closed with respect to unions, as in Assumption 1 (iii). Given our interpretation of the collection $\mathcal{N}$, an investor disregards differences between elements of $\mathfrak{B}(\mathcal{F})$ which are equal up to negligibility and is thus led to consider the quotient space $\mathfrak{B}(\mathcal{F}, \mathcal{N})$ of equivalence classes of $\mathfrak{B}(\mathcal{F})$ : let $\varkappa: \mathfrak{B}(\mathcal{F}) \rightarrow \mathfrak{B}(\mathcal{F}, \mathcal{N})$ be the quotient map. $\mathfrak{B}(\mathcal{F}, \mathcal{N})$ has therefore a linear structure only if $=$ u.n. is an equivalence relation, i.e. ultimately if Assumption 1(iii) holds.

It is rather clear that whenever $\mathcal{N}$ coincides with the collection $\mathcal{N}_{Q}$ of null sets generated by some $Q \in \mathbb{P}(\mathcal{F})$ then $\mathfrak{B}\left(\mathcal{F}, \mathcal{N}_{Q}\right)=L^{\infty}(Q)$; another special case is $\mathcal{N}=\{\varnothing\}$ where we get $\mathfrak{B}(\mathcal{F},\{\varnothing\})=\mathfrak{B}(\mathcal{F})$.

$b a(\mathcal{F}, \mathcal{N})$ denotes the set of finitely additive measures on $\mathcal{F}$ which vanish on $\mathcal{N}$; for the lattice theoretic terminology adopted in the next theorem see [2].

Theorem 1. $\mathfrak{B}(\mathcal{F}, \mathcal{N})$ is a Banach lattice under the norm $\|\varkappa(f)\|_{\mathfrak{B}(\mathcal{F}, \mathcal{N})}=\inf _{N \in \mathcal{N}}\left\|f N^{c}\right\|_{\mathfrak{B}(\mathcal{F})}$ and the map $\varkappa$ a lattice isomorphism. Moreover, the topological dual $\mathfrak{B}(\mathcal{F}, \mathcal{N})^{*}$ of $\mathfrak{B}(\mathcal{F}, \mathcal{N})$ and ba $(\mathcal{F}, \mathcal{N})$ are isometrically isomorphic via the equation

$$
\phi(\varkappa(f))=\int f d \mu
$$

where $\phi \in \mathfrak{B}(\mathcal{F}, \mathcal{N})^{*}$ and $\mu \in b a(\mathcal{F}, \mathcal{N})$.

Proof. By Lemma 1, two elements $f, g$ of $\mathfrak{B}(\mathcal{F})$ are equal up to negligibility if and only if $f-g \in \overline{\mathfrak{L}(\mathcal{N})}$ so that $\mathfrak{B}(\mathcal{F}, \mathcal{N})$ actually coincides with the quotient space $\mathfrak{B}(\mathcal{F}) \backslash \overline{\mathfrak{L}(\mathcal{N})}$ which is known to be a Banach space under the norm

$$
\|\| \varkappa(f) \|\left.\right|_{\mathfrak{B}(\mathcal{F}, \mathcal{N})}=\inf \left\{\|f-u\|_{\mathfrak{B}(\mathcal{F})}: u \in \overline{\mathfrak{L}(\mathcal{N})}\right\}
$$

[31, 1.7.7, p. 53]. If $N \in \mathcal{N}$ then $f N \in \overline{\mathfrak{L}(\mathcal{N})}$ and $\left\|f N^{c}\right\|_{\mathfrak{B}(\mathcal{F})}=\|f-f N\|_{\mathfrak{B}(\mathcal{F})}$; if $u \in \mathfrak{L}(\mathcal{N})$ then $N_{u}=\{|u|>0\} \in \mathcal{N}$ and $\|f-u\|_{\mathfrak{B}(\mathcal{F})} \geq\left\|f N_{u}^{c}\right\|_{\mathfrak{B}(\mathcal{F})}$. We conclude (by the continuity of the norm) \|\|$\varkappa(f) \mid\left\|_{\mathfrak{B}(\mathcal{F}, \mathcal{N})}=\right\| \varkappa(f) \|_{\mathfrak{B}(\mathcal{F}, \mathcal{N})}$. Let $f, g \in \mathfrak{B}(\mathcal{F}), g \geq 0$ u.n. and $g \geq f$ u.n.. Then $g^{+}=g$ u.n. and $g^{+} \geq f$ u.n. implies $\left\{f^{+}-g^{+} \geq \eta\right\}=\left\{f-g^{+} \geq \eta\right\} \in \mathcal{N}$, that is $g^{+} \geq f^{+}$u.n.; $f^{+} \geq f$ u.n. is also clear from Lemma 1. We thus conclude that $f^{+}$is the least element in the set $\{g \in \mathfrak{B}(\mathcal{F}): g \geq f$ u.n., $g \geq 0$ u.n. $\}$ i.e. that $\varkappa(f \vee 0)=\varkappa(f) \vee \varkappa(0)$ and, by linearity, that $\varkappa(f \vee g)=\varkappa(f) \vee \varkappa(g)$. Therefore $\mathfrak{B}(\mathcal{F}, \mathcal{N})$ is a vector lattice and $\varkappa$ commutes with the lattice operations, i.e. it is a lattice isomorphism. $|\varkappa(f)| \geq|\varkappa(g)|$ is then equivalent to $|f| \geq|g|$ u.n. i.e. $\{|f|+\eta \leq|g|\} \in \mathcal{N}$. But then

$$
\|\varkappa(f)\|_{\mathfrak{B}(\mathcal{F}, \mathcal{N})}=\inf _{N \in \mathcal{N}}\left\||f| N^{c}\{|f|+\eta>|g|\}\right\|_{\mathfrak{B}(\mathcal{F})} \geq \inf _{N \in \mathcal{N}}\left\||g| N^{c}\right\|_{\mathfrak{B}(\mathcal{F})}-\eta=\|\varkappa(g)\|_{\mathfrak{B}(\mathcal{F}, \mathcal{N})}-\eta
$$

so $\|\varkappa(f)\|_{\mathfrak{B}(\mathcal{F}, \mathcal{N})} \geq\|\varkappa(g)\|_{\mathfrak{B}(\mathcal{F}, \mathcal{N})}$ and $\mathfrak{B}(\mathcal{F}, \mathcal{N})$ is a Banach lattice.

If $\mu \in b a(\mathcal{F}, \mathcal{N})$ then the right hand side of $(2.1)$ defines a linear functional over $\mathfrak{B}(\mathcal{F}, \mathcal{N})$ and the inequality

$$
\left|\int f d \mu\right|=\inf _{N \in \mathcal{N}}\left|\int f N^{c} d \mu\right| \leq \inf _{N \in \mathcal{N}}\left\|f N^{c}\right\|_{\mathfrak{B}(\mathcal{F})}\|\mu\|=\|f\|_{\mathfrak{B}(\mathcal{F}, \mathcal{N})}\|\mu\|
$$


proves that it is bounded and of norm less than $\|\mu\|$. If $\phi \in \mathfrak{B}(\mathcal{F}, \mathcal{N})^{*}$, then $\varkappa^{*} \phi \in \mathfrak{B}(\mathcal{F})^{*}$ and is therefore isometrically isomorphic to some $\mu \in b a(\mathcal{F})$ satisfying (2.1), and thus vanishing on $\mathcal{N}$. $\left\|\varkappa^{*}\right\|=1$ implies $\|\mu\|=\left\|\varkappa^{*} \phi\right\| \leq\|\phi\|$. We conclude that $\|\mu\|=\|\phi\|$.

Thanks to Theorem 1 we are entitled to speak of the elements of $\mathfrak{B}(\mathcal{F}, \mathcal{N})$ as if they were functions rather than classes of equivalent functions so that the same symbol $f$ will be used to denote an element of $\mathfrak{B}(\mathcal{F})$ and the element of $\mathfrak{B}(\mathcal{F}, \mathcal{N})$ associated to it. As for order, $f \geq g$ will be used for $\mathfrak{B}(\mathcal{F})$ while $f \geq g$ u.n. for $\mathfrak{B}(\mathcal{F}, \mathcal{N}) ;\|\cdot\|$ will be used in place of $\|\cdot\|_{\mathfrak{B}(\mathcal{F}, \mathcal{N})}$. We thus define the positive cones

$$
\mathfrak{B}(\mathcal{F}, \mathcal{N})_{+}=\{f \in \mathfrak{B}(\mathcal{F}, \mathcal{N}): f \geq 0 \text { u.n. }\} \text { and } \mathfrak{B}(\mathcal{F}, \mathcal{N})_{++}=\{f \in \mathfrak{B}(\mathcal{F}, \mathcal{N}): f>0 \text { u.n. }\}
$$

A natural question, to which we shall often return later, is whether negligibility, whatever its source, may be reconciled with probability. Let $\mathbb{P}(\mathcal{F}, \mathcal{N})$ denote the subset of $\mathbb{P}(\mathcal{F})$ consisting of probability measures $P$ on $\mathcal{F}$ such that $P(N)=0$ for each $N \in \mathcal{N} ;$ let $\mathbb{P}_{b a}(\mathcal{F}, \mathcal{N})$ be defined likewise.

Theorem 2. $\mathbb{P}_{b a}(\mathcal{F}, \mathcal{N}) \neq \varnothing$ while the following are equivalent:

a. $\mathbb{P}(\mathcal{F}, \mathcal{N}) \neq \varnothing ;$

b. there exists $Q \in \mathbb{P}(\mathcal{F})$ such that for any increasing sequence $\left\langle F_{n}\right\rangle_{n \in \mathbb{N}}$ of sets in $\mathcal{N}$

$$
\lim _{n} Q\left(F_{n}^{c}\right)>0
$$

Proof. $\Omega$ is an inner point for $\mathfrak{B}(\mathcal{F}, \mathcal{N})_{++}$as $\Omega \in \mathfrak{B}(\mathcal{F}, \mathcal{N})_{++}$(see remark 1) and $\|f-\Omega\|<\eta$ implies $\{f<1-\eta\} \in \mathcal{N}$. The linear functional $\phi$ separating the convex sets $\mathfrak{B}(\mathcal{F}, \mathcal{N})_{++}$and $\{0\}$ will therefore be bounded and non trivial, i.e. $\phi(\Omega)>0$, and such that $\phi\left[\mathfrak{B}(\mathcal{F}, \mathcal{N})_{++}\right] \geq \phi(0)=0$. By Theorem $1 \phi$ is associated to some $m \in b a(\mathcal{F}, \mathcal{N})_{+}$that can be normalized so that $m(\Omega)=1$.

Let $\mathcal{R}(\mathcal{N})$ be the $\sigma$ ring generated by the collection $\mathcal{N}$ and $\mathcal{R}(\mathcal{N})^{\perp}=\left\{F \subset \Omega: F^{c} \in \mathcal{R}(\mathcal{N})\right\}$. By Assumption 1 it follows that $\mathcal{R}(\mathcal{N})=\left\{\bigcup_{k \in \mathbb{N}} N_{k}: N_{k} \in \mathcal{N}, k \in \mathbb{N}\right\} ;$ it is well known that $\sigma \mathcal{N}=\mathcal{R}(\mathcal{N}) \cup \mathcal{R}(\mathcal{N})^{\perp}$. Assume that (b) holds, denote by $\mathcal{N}_{Q}$ the collection of $Q$ null sets and let $\overline{\mathcal{N}}=\left\{F \cup G: F \in \mathcal{N}, G \in \mathcal{N}_{Q}\right\}$. $\mathcal{R}(\overline{\mathcal{N}})$ satisfies the properties listed in Assumption 1 so that, by the first claim of this Lemma, there exists $\lambda \in \mathbb{P}_{b a}(\sigma \overline{\mathcal{N}}, \mathcal{R}(\overline{\mathcal{N}}))$. If $F, G \in \mathcal{R}(\overline{\mathcal{N}})^{\perp}$ are disjoint, then $\Omega \in \mathcal{R}(\overline{\mathcal{N}})$ a contradiction; if $\left\langle F_{n}\right\rangle_{n \in \mathbb{N}}$ is a disjoint sequence of $\sigma \overline{\mathcal{N}}$ measurable sets, then it has at most one element in $\mathcal{R}(\overline{\mathcal{N}})^{\perp}$, say $F_{1}$, while $\bigcup_{n>1} F_{n} \in \mathcal{R}(\overline{\mathcal{N}})$. Then,

$$
\lambda\left(\bigcup_{n} F_{n}\right)=\lambda\left(F_{1}\right)+\lambda\left(\bigcup_{n>1} F_{n}\right)=\lambda\left(F_{1}\right)=\lambda\left(F_{1}\right)+\sum_{n>1} \lambda\left(F_{n}\right)
$$

Since each two versions of $Q(F \mid \sigma \mathcal{N})$ coincide outside some $F \in \overline{\mathcal{N}}$, there is no ambiguity defining $P(F)=$ $\lambda(Q(F \mid \sigma \mathcal{N})) . P$ is positive and $P(\Omega)=1$; furthermore, $P$ vanishes on $\mathcal{N}$. If $\left\langle F_{n}\right\rangle_{n \in \mathbb{N}}$ is a disjoint sequence of $\mathcal{F}$ measurable sets, then $Q\left(\bigcup_{n} F_{n} \mid \sigma \mathcal{N}\right)=\sum_{n} Q\left(F_{n} \mid \sigma \mathcal{N}\right)$ up to a $\lambda$ null set and since $\lambda \in \mathbb{P}(\sigma \overline{\mathcal{N}})$,

$$
P\left(\bigcup_{n} F_{n}\right)=\lambda\left(Q\left(\bigcup_{n} F_{n} \mid \sigma \mathcal{N}\right)\right)=\lambda\left(\sum_{n} Q\left(F_{n} \mid \sigma \mathcal{N}\right)\right)=\sum_{n} \lambda\left(Q\left(F_{n} \mid \sigma \mathcal{N}\right)\right)=\sum_{n} P\left(F_{n}\right)
$$

(a) follows. The converse is obvious.

It is therefore always possible to find some finitely additive probability which is compatible with $\mathcal{N}$ in the above sense while it need not if countable additivity is required: if, e.g., $\Omega=\bigcup_{n} F_{n}$ and $F_{n} \in \mathcal{N} n \geq 1$ then any $m \in b a(\mathcal{F}, \mathcal{N})$ is purely finitely additive. Condition (2.3) clearly rules this case out and all it requires is the existence of a measure not in blatant contrast with the interpretation of $\mathcal{N}$ as a collection of null sets. 
2.3. Asset Returns and Trading Strategies. We denote by $\mathbb{K}$ the set of admissible, discounted returns ${ }^{4}$ and assume throughout the paper the following:

Assumption 2. $\mathbb{K}$ is a linear subspace of $\mathfrak{B}(\tilde{\mathcal{F}})$ consisting of càdlàg processes $K$ such that

(i). $K_{t}$ is adapted and $K_{0}=0$;

(ii). there exists $T \in \mathbb{R}_{+}$such that $K=K^{T}$;

(iii). $\theta . K \in \mathbb{K}$ whenever $\theta$ is of the form $\left.\left.\left.\left.\theta=\sum_{m=1}^{M} \theta_{m}\right]\right] \tau_{m}, \tau_{m+1}\right]\right]$ where $\tau_{m} \in \mathcal{T}$ and $\theta_{m} \in \mathfrak{L}\left(\mathcal{F}_{\tau_{m}}\right)$, $m=1, \ldots, M$.

We also define

$$
\mathcal{K}=\left\{k \in \mathfrak{B}(\mathcal{F}, \mathcal{N}): k=K_{\infty} \text { u.n. for some } K \in \mathbb{K}\right\}
$$

and

$$
\mathcal{C}=\mathcal{K}-\mathfrak{B}(\mathcal{F}, \mathcal{N})_{+}
$$

Assumption 2 seems to us a reasonable approximation to the way real markets actually work on three grounds. First, the strategies considered do not imply a life commitment for investors. Second, trading only involves a finite number of transactions: the cost of trading - which may either consist of explicit transaction fees or be simply implicit in information processing - is then certain and reasonable. Eventually, each transaction is contingent on a finite number of scenarios, a feature making the actual implementation of the investment strategy realistically simple; it also captures the increasing importance of scenario analysis in the investment industry (see [30]). Observe that pathological situations which are of concern in the traditional approach - like so called "doubling strategies" - do not arise here. The linear structure of $\mathbb{K}$ signifies, as usual, that trading is unrestricted and that short positions are allowed. It is possible that, at the price of additional complications, this assumption may be relaxed. Preventing possibly unbounded losses in the absence of an exogenous probability measure translates quite naturally (or, rather, unavoidably) into the requirement that return processes be lower bounded. Given that this constraint should apply no matter the sign of the position taken, long or short, returns will be modeled as bounded functions on $\Omega \times \mathbb{R}_{+}$.

A market in which the diversification of risk is unrestricted would allow investors to diversify their portfolios at will across admissible investment projects, provided their resulting position does not imply the possibility of unbounded losses. In such a market portfolio returns would be described by the set

$$
\mathbb{K}_{\sigma}=\left\{\sum_{n} K^{n}: K^{n} \in \mathbb{K}, n \geq 1, \sum_{n}\left\|K^{n-}\right\|_{\mathfrak{B}(\tilde{\mathcal{F}})}<\infty\right\}
$$

Let

$$
\mathcal{C}_{\sigma}=\left\{k \in \mathfrak{B}(\mathcal{F}, \mathcal{N}): k=K_{\infty} \wedge r \text { u.n. for some } K \in \mathbb{K}_{\sigma} \text { and } r>0\right\}
$$

A time honored issue in the theory of finance is that of completeness of markets, introduced in [24], [25] and [26] (see also [6] and [7] for a different approach). In our model completeness is defined as follows:

Definition 3. The financial markets described by $\mathbb{K}$ are complete if for every $f \in \mathfrak{B}(\mathcal{F}, \mathcal{N})$ there exists $\gamma(f) \in \mathbb{R}$ such that $f-\gamma(f) \in \mathcal{K}$.

\footnotetext{
${ }^{4}$ The counterpart to $\mathbb{K}$ in the traditional approach is the set $\{H . S: H$ admissible $\}$, where $S$ denotes the semimartingale price process and the definition of admissibility is as in [16, definition 2.7, p. 472].
} 
Remark 2. At times the qualification "over finite horizon" is appended to properties - such as market completeness - which involve $\mathfrak{B}(\mathcal{F}, \mathcal{N})$. In doing so we mean that the corresponding definition is assumed to hold with $\mathfrak{B}(\mathcal{F}, \mathcal{N})$ replaced by $\mathfrak{B}\left(\mathcal{F}_{t}, \mathcal{N}\right)$, for all $t \in \mathbb{R}_{+}$.

\section{Arbitrage and The Pricing Measure}

Any sensible model of financial markets should exclude the existence of free money as, in the absence of restrictions to trade, this would contrast with the existence of equilibrium. In the context of the present setting, an arbitrage opportunity occurs whenever there exists an admissible investment yielding a return which, in discounted terms, is strictly positive up to negligibility. This definition of an arbitrage opportunity is therefore compatible with any system of preferences according to which $f$ is strictly preferred to $g$ whenever $f-g \in \mathfrak{B}(\mathcal{F}, \mathcal{N})_{++}$, no other property such as continuity or convexity being invoked. Remark that preferences of this sort may indeed be considered as an exemplification of over confidence as the circumstance $f>g$ u.n. does not exclude that events such as $\{f<g-\eta\}$ could occur but simply that these will not be considered by the decision maker. More formally we require

$$
\mathcal{K} \cap \mathfrak{B}(\mathcal{F}, \mathcal{N})_{+}=\{0\}
$$

Many versions of the above condition appear in the literature, all considerably more restrictive than (3.1). Not only is a richer structure of asset returns assumed, but the concept of an arbitrage opportunity is often conveniently reinforced into that of a free lunch (see [11], [12] and the seminal paper by Kreps [29], for a discussion). The formal definition of the absence of free lunches in our setting is

$$
\overline{\mathcal{C}} \cap \mathfrak{B}(\mathcal{F}, \mathcal{N})_{+}=\{0\}
$$

(the upper bar denotes closure in the norm topology of $\mathfrak{B}(\mathcal{F}, \mathcal{N})$ ).

Let us introduce the following quantities, where $k \in \mathcal{K}$ and $f \in \mathbb{R}^{\Omega}$ (and conventionally inf $\varnothing=\infty$ and $\sup \varnothing=-\infty)$

$$
\bar{\alpha}_{k}(f)=\inf \{a \in \mathbb{R}: a \geq k+f \text { u.n. }\} \quad \underline{\alpha}_{k}(f)=\sup \{a \in \mathbb{R}: a \leq k+f \text { u.n. }\}
$$

and

$$
\bar{\alpha}_{\mathcal{K}}(f)=\inf _{k \in \mathcal{K}} \bar{\alpha}_{k}(f) \quad \underline{\alpha}_{\mathcal{K}}(f)=\sup _{k \in \mathcal{K}} \underline{\alpha}_{k}(f)
$$

It is obvious that $\bar{\alpha}_{\mathcal{K}}$ is subadditive and that $\bar{\alpha}_{\mathcal{K}}(r f)=r \bar{\alpha}_{\mathcal{K}}(f)$ for $r>0$ while $\bar{\alpha}_{\mathcal{K}}(r f)=r \underline{\alpha}_{\mathcal{K}}(f)$ for $r<0$. Remark also that, by (3.3), $\eta>0$ implies that $\bar{\alpha}_{k}(f)+\eta \geq k+f$ u.n. i.e. $\left\{\bar{\alpha}_{k}(f)+2 \eta \leq k+f\right\} \in \mathcal{N}$. In other words

$$
\bar{\alpha}_{k}(f) \geq k+f \geq \underline{\alpha}_{k}(f) \quad \text { u.n. }
$$

We define also the set of pricing measures

$$
\mathcal{M}(\mathcal{C})=\left\{m \in \mathbb{P}_{b a}(\mathcal{F}, \mathcal{N}): m[\mathcal{C}] \leq 0\right\}
$$

Theorem 3. If the market is free of arbitrage opportunities then there exists a pricing measure $m \in \mathcal{M}(\mathcal{C})$. If markets are complete (resp. complete over finite horizons) and $F \in \mathcal{F}$ (resp. $F \in \bigcup_{t \in \mathbb{R}_{t}} \mathcal{F}_{t}$ ), then $m(F)=0$ if and only if $F \in \mathcal{N}$. 
Proof. As noted already, $\Omega$ is an internal point of $\mathfrak{B}(\mathcal{F}, \mathcal{N})_{++}$, there exists a non trivial, continuous linear functional $\phi$ that separates $\mathfrak{B}(\mathcal{F}, \mathcal{N})_{++}$and $\mathcal{K}$. Since $\phi[\mathcal{K}]$ is a linear space and $\phi\left[\mathfrak{B}(\mathcal{F}, \mathcal{N})_{++}\right]$a convex cone, $\phi[\mathcal{K}] \cap \phi\left[\mathfrak{B}(\mathcal{F}, \mathcal{N})_{++}\right] \subset\{0\}$ implies that, up to a change of sign, $\phi[\mathcal{K}]=0 \leq \phi\left[\mathfrak{B}(\mathcal{F}, \mathcal{N})_{++}\right]$and $\phi(\Omega)>0$. By Theorem 1 and normalization we may represent $\phi$ via $m \in \mathbb{P}_{b a}(\mathcal{F}, \mathcal{N})$. If $F \in \mathfrak{B}\left(\mathcal{F}_{t}, \mathcal{N}\right)_{+}$for some $t \in \mathbb{R}_{+}$and markets are complete over finite horizons or $F \in \mathfrak{B}\left(\mathcal{F}_{t}, \mathcal{N}\right)_{+}$for some $t \in \mathbb{R}_{+}$and markets are complete, then $F-\gamma(F) \in \mathcal{K}$ for some $\gamma(F)$. Then necessarily $\gamma(F)=m(F)$ so that $m(F)=0$ implies $F \in \mathcal{K} \cap \mathfrak{B}(\mathcal{F}, \mathcal{N})_{+}$which contradicts $(3.1)$ unless $F \in \mathcal{N}$.

In the traditional setting, the existence of a pricing measure (often termed variously a risk neutral or a martingale measure) gets along with the existence of a state price density process or stochastic discount factor, e.g. [21, p. 47]. Such process, often obtained from the first order conditions for portfolio choice, has a crucial role in financial modelling to the extent that it is common practice to simply assume its existence. In section 5 we shall obtain a process strictly related to the state price process. In the next example, borrowed from [16, p. 510], we show a fairly natural connection between state price processes and finitely additive pricing measures.

Example 1. Let $(\Omega, \mathcal{F}, P)$ be a standard probability space, $\mathcal{N}=\mathcal{N}_{P}$ and let $B$ be Brownian motion over $[0,1]$ and $\left(\mathcal{F}_{t}: t \in[0,1]\right)$ its natural filtration. Define the processes

$$
S_{t}=-2 \sqrt{1-t}+B_{t}
$$

and

$$
Z_{t}=\mathcal{E}\left(-\int_{0}^{t} \frac{1}{\sqrt{1-s}} d B_{s}\right), \quad 0 \leq t<1 \text { and } Z_{t}=0, \quad t=1
$$

$Z$ is a strictly positive martingale which converges $P$ a.s. to 0 [35, proposition VIII.1.15, p. 332 and exercise VIII.1.29, p. 335]: it fails thus to be uniformly integrable. Let $\Theta$ consist of simple processes stopped by some stopping time $T_{n}=\inf \left\{t \in[0,1]:\left|B_{t}\right|+\left|Z_{t}\right| \geq 2^{n}\right\} \wedge\left(1-2^{-n}\right)$ and $\mathbb{K}=\{\theta . S: \theta \in \Theta\}$. It is clear that $\mathbb{K}$ satisfies Assumption 2 above; moreover, $Z K$ is a martingale for all $K \in \mathbb{K}$. Define

$$
\mu(F)=\operatorname{LIM}_{n} P\left(Z_{1-2^{-n}} F\right), \quad F \in \mathcal{F}
$$

where LIM denotes the so called Banach limit introduced in [1, p. 23]. It is then pretty obvious that if $K \in \mathbb{K}$ then there is $t_{0}$ such that $K_{\infty}=K_{t_{0}}$ so that

$$
\mu\left(K_{\infty}\right)=\operatorname{LIM}_{n} P\left(Z_{1-2^{-n}} K_{\infty}\right)=P\left(Z_{t_{0}} K_{t_{0}}\right)=0
$$

Thus $\mu \in \mathcal{M}(\mathcal{C})$. Moreover, there is no arbitrage since $P\left(Z_{t} K_{\infty}\right)=0$ holding for $t$ sufficiently large and $P\left(K_{\infty} \geq 0\right)=1$ imply $P\left(Z_{t}\left\{K_{\infty}>0\right\}\right)=0$ i.e. $P\left(K_{\infty}>0\right) \leq P\left(Z_{t}=0\right)=0$. The construction (3.8) was used in [10] to illustrate the role of asset bubbles.

A natural question is under which additional assumptions may countable additivity be established. The following provides an answer under quite special conditions

Theorem 4. There exists a countably additive pricing measure $m_{\sigma}$ provided the following holds:

$(a) . \mathcal{N}$ is closed under countable unions;

(b). for any sequence $\left\langle f_{n}\right\rangle_{n \in \mathbb{N}}$ in $\mathfrak{B}(\mathcal{F})_{+}$such $\sum_{n} f_{n} \in \mathfrak{B}(\mathcal{F})_{+}, \sum_{n} \bar{\alpha}_{\mathcal{K}}\left(f_{n}\right)<\infty$;

(c). $\mathcal{C}_{\sigma} \cap \mathfrak{B}(\mathcal{F}, \mathcal{N})_{+}=\{0\}$ 
Theorem 4 suggests that countable additivity of the pricing measure $m$ is related to a sufficient degree of sophistication in assessing uncertainty (condition $(a)$ ) as well as in the diversification of portfolio (condition $(c)$ ) and the efficiency of markets in the hedging of risks (condition $(b)$ ). Theorem 4 contributes to the view that countable additivity is more an artifact of the theory than a property of markets.

\section{An Explicit Representations.}

Although finitely additive probabilities exist under fairly general conditions, their analytical tractability raises problems, especially for convergence theorems. In a companion paper [9] we developed some results that contribute considerably to the developments of the following sections. We recall here such results, partly offering new proofs, and develop further ones (the proofs appear in the Appendix).

4.1. The Representing Measure. In the theory of finitely additive measures the decomposition of Yosida and Hewitt [44, theorem 1.24, p. 52] is probably the best known and most useful result. We shall use the following generalization proved in [9, theorem 1]:

Lemma 2. Let $v \in b a(\mathcal{F}), \mathcal{G}$ be a sub $\sigma$ algebra of $\mathcal{F}$ and $v_{\mathcal{G}}$ the restriction $v \mid \mathcal{G}$ of $v$ to $\mathcal{G}$. There exists a unique way of writing

$$
v_{\mathcal{G}}=v_{\mathcal{G}}^{e}+v_{\mathcal{G}}^{p}
$$

with $v_{\mathcal{G}}^{e}, v_{\mathcal{G}}^{p} \in b a(\mathcal{G})$, where $v_{\mathcal{G}}^{e}$ admits a countably additive extension to $\mathcal{F}$ and any norm preserving extension of $v_{\mathcal{G}}^{p}$ to $\mathcal{F}$ is purely finitely additive. Furthermore:

(i). if $v \geq 0$ then $v_{\mathcal{G}}^{e}, v_{\mathcal{G}}^{p} \geq 0$;

(ii). $v_{\mathcal{G}}^{p}$ is orthogonal to any $P \in c a(\mathcal{F})_{+}$i.e. for each $\epsilon>0$ there is $G \in \mathcal{G}$ such that $v_{\mathcal{G}}^{p}(G)=0$ and $P\left(G^{c}\right)<\epsilon$;

(iii) if $\mathcal{H}$ is a sub $\sigma$ algebra of $\mathcal{G}$ and $v_{\mathcal{H}}=v_{\mathcal{H}}^{e}+v_{\mathcal{H}}^{p}$ the decomposition (4.1), then $v_{\mathcal{H}}^{e} \geq v_{\mathcal{G}}^{e} \mid \mathcal{H}$ and $v_{\mathcal{H}}^{p} \leq v_{\mathcal{G}}^{p} \mid \mathcal{H}$

In the case $\mathcal{G}=\mathcal{F}$ this decomposition coincides with that of Yosida and Hewitt.

The application of Lemma 2 to our model will be by setting $v=m$ and $\mathcal{G}=\mathcal{F}_{\tau}$ for some $\tau \in \mathcal{T}-$ in which case we write $m_{\tau}$ rather than $m_{\mathcal{F}_{\tau}}$. For reasons that will soon become clear we shall rather be interested in the right continuous versions $m_{\tau+}^{e}, m_{\tau+}^{p} \in b a\left(\mathcal{F}_{\tau}\right)$ of the above decomposants defined implicitly by setting

$$
m_{\tau+}^{e}(F)=\lim _{n} m_{\tau+2^{-n}}^{e}(F) \text { and } m_{\tau+}^{p}(F)=\lim _{n} m_{\tau+2^{-n}}^{p}(F), \quad F \in \mathcal{F}_{\tau}
$$

Remark that $m_{\tau+}^{e} \leq m_{\tau}^{e}-$ so that $m_{\tau+}^{e}$ admits a countably additive extension to $\mathcal{F}-$ and that $m_{\tau}=$ $m_{\tau+}^{e}+m_{\tau+}^{p}$. Moreover when $\sigma \in \mathcal{T}$ satisfies $\sigma \leq \tau$ then

$$
m_{\tau+}^{p}\left|\mathcal{F}_{\sigma}=m_{\sigma}^{p}+\left(m_{\sigma}^{e}-m_{\tau+}^{e}\right)\right| \mathcal{F}_{\sigma}
$$

It is immediate that (4.3) coincides with the decomposition (4.1) as applied to $m_{\tau+}^{p} \mid \mathcal{F}_{\sigma}$.

Example 2. In the context of Example 1 it is clear that the restriction $\mu_{t}$ of the pricing measure $\mu$ defined in (3.8) to $\mathcal{F}_{t}$ admits a countably additive extension to $\mathcal{F}_{1}$ defined implicitly by letting

$$
\bar{\mu}_{t}(F)=P\left(Z_{t} F\right), \quad F \in \mathcal{F}_{1}
$$


so that $\mu_{t}^{p}=0$. However, $\mu^{e}=0$. In fact let $Z_{1}^{*}=\sup _{0 \leq t \leq 1} Z_{t}, F_{n}=\left\{n-1<Z_{1}^{*} \leq n\right\}$ for each $n \geq 1$ and $F_{0}=\bigcap_{n} F_{n}^{c}$. Then $\mu\left(F_{0}\right)=0$ follows from $P\left(F_{0}\right)=0$ and $\mu^{e} \ll P$ while, as $Z_{t}$ converges $P$ a.s. to 0

$$
\mu\left(F_{n}\right)=\operatorname{LIM}_{n} P\left(Z_{1-2^{-n}} F_{n}\right) \leq \operatorname{LIM}_{n} P\left(Z_{1-2^{-n}}\left\{Z_{1}^{*} \leq n\right\}\right)=P\left(Z_{1}\left\{Z_{1}^{*} \leq n\right\}\right)=0
$$

But then, $\mu^{e}(\Omega)=\sum_{n \geq 0} \mu^{e}\left(F_{n}\right) \leq \sum_{n \geq 0} \mu\left(F_{n}\right)=0$. We conclude that $\mu$ is purely finitely additive. In [10] it is shown that $\mu$ is countably additive if and only if $Z$ is uniformly integrable.

The component $m_{t}^{e}$ - or rather its countably additive extension $\bar{m}_{t}^{e}$ - may be viewed as a fully additive assessment of randomness implicit in $m$ given the information $\mathcal{F}_{t}$ available at time $t$. The arrival of new information over time modifies such assessment as the decomposition (4.1) depends on the underlying information structure. In particular, by Lemma 2(iii) the larger is the underlying information set the "smaller" is the part of $m$ which admits a countable additive extension, thus accounting for the increasing difficulty of recovering countable additivity as information increases. The question therefore arises whether it is possible to extract from the collection $\left\{\bar{m}_{t}^{e}: t \in \mathbb{R}_{+}\right\}$a global perspective $P$ on $\mathcal{F}$ not contradicting the inference $m_{t}^{e}$ made at each point in time. Although different, sensible criteria could be considered in order to judge whether $P$ contrasts with $m_{t}^{e}$ or not, a clear contradiction definitely exists between these two measures whenever, for some $F \in \mathcal{F}_{t}, m_{t}^{e}(F)>0$ but $P(F)=0$ : it may well be that events that are locally null have a positive global probability, but the opposite would indeed imply that the global assessment expressed by $P$ implicitly disproves the one embodied in $m_{t}^{e}$. The following result provides a positive answer to the above question.

Theorem 5. There exist $P \in \mathbb{P}(\mathcal{F})$ such that $m_{t}^{e} \ll P \mid \mathcal{F}_{t}$ and $m_{\tau+}^{e} \ll P \mid \mathcal{F}_{\tau}$ for each $t \in \mathbb{R}_{+}$and $\tau \in \mathcal{T}$ such that $\tau<\infty$. Moreover,

(i). If $m \in \mathbb{P}_{b a}(\mathcal{F}, \mathcal{N})$ and $\mathbb{P}(\mathcal{F}, \mathcal{N}) \neq \varnothing$, then we may choose $P \in \mathbb{P}(\mathcal{F}, \mathcal{N})$;

(ii). If $X_{\tau}^{P}$ is the Radon Nikodym derivative of $m_{\tau+}^{e}$ with respect to $P \mid \mathcal{F}_{\tau}$, then $X^{P}$ is a positive, right continuous $P$ supermartingale.

A probability such as $P$ in Theorem 5 will be termed a representing measure for $m^{5}$ and we denote

$$
\mathbb{P}(m)=\left\{P \in \mathbb{P}(\mathcal{F}): m_{t}^{e} \ll P \mid \mathcal{F}_{t}, \quad t \in \mathbb{R}_{+} \text {and } m_{\tau+}^{e} \ll P \mid \mathcal{F}_{\tau}, \tau \in \mathcal{T}, \tau<\infty\right\}
$$

while $\mathbb{P}(m, \mathcal{N})=\mathbb{P}(m) \cap \mathbb{P}(\mathcal{F}, \mathcal{N})$. The "endogenous" nature of each $P \in \mathbb{P}(m)$ is such that it will in general depend partly on subjective elements, via the collection $\mathcal{N}$, and partly on the structure of markets, via $\mathbb{K}$. Despite the multiplicity of elements in $\mathbb{P}(m)$, in the following sections we will treat $P \in \mathbb{P}(m)$ and $X^{P}$ as fixed - we shall therefore write more simply $X$.

4.2. Conditional Expectation. A straightforward implication of the existence of a pricing measure in the traditional setting is that investment returns obey a martingale restriction with respect to it. This is also of fundamental importance in order to establish a clear, backward pricing rule. These conclusions are unlikely to carry over to our model as conditional expectation is not available with respect to finitely additive probability. The following proposition (that also appears in [9] but we offer here an entirely different proof) introduces a concept of conditional expectation for finitely additive probabilities which is suitable for our purposes. For ease of terminology, we will refer to such operator as conditional expectation although it may fail to satisfy the law of iterated expectation.

\footnotetext{
${ }^{5}$ Curiously enough, the term representing measure was used by Delbaen and Schachermayer [16, p. 495] with much the same meaning as the term pricing measure is used here.
} 
Proposition 1. Let $v \in b a(\mathcal{F})_{+}, \mathcal{G} \subset \mathcal{F}$ be a $\sigma$ algebra and $\gamma+\lambda$ be an orthogonal decomposition of $v_{\mathcal{G}}$, with $\gamma \in c a(\mathcal{G})_{+}$and $\lambda \in b a(\mathcal{G})_{+}$. Define

$$
\mathcal{I}_{\lambda}=\{F \in \mathcal{G}: \lambda(F)=0\}
$$

Then, for each $f \in L^{1}(v)$ there exists a unique $v\left(f \mid \mathcal{I}_{\lambda}\right) \in L^{1}(\gamma)$ such that

$$
v(f I)=v\left(v\left(f \mid \mathcal{I}_{\lambda}\right) I\right)=\gamma\left(v\left(f \mid \mathcal{I}_{\lambda}\right) I\right)
$$

for each $I \in \mathcal{I}_{\lambda}$ and that for any $G \in \mathcal{G}$

$$
v\left(f G \mid \mathcal{I}_{\lambda}\right)=v\left(f \mid \mathcal{I}_{\lambda}\right) G
$$

The mapping $v\left(\cdot \mid \mathcal{I}_{\lambda}\right): L^{1}(v) \rightarrow L^{1}(\gamma)$ is a positive, linear operator of norm one.

This result is of special importance as it allows to apply conditioning to the pricing measure $m \in \mathcal{M}(\mathcal{C})$, in particular with reference to decomposition (4.1). For each $\sigma \in \mathcal{T}$ we therefore agree to write $\mathcal{I}_{\sigma}$ as short for $\mathcal{I}_{m_{\sigma}^{p}}$ and we deduce from Proposition 1 the existence of the conditional expectation $m_{\tau}\left(f \mid \mathcal{I}_{\sigma}\right)$ whenever $\tau \in \mathcal{T}$ and $\sigma \leq \tau$. Yet another application is based on decomposition (4.3) establishing the existence of the conditional expectation $m_{\tau+}^{p}\left(f \mid \mathcal{I}_{\sigma}\right) \in L^{1}\left(\Omega, \mathcal{F}_{\sigma}, m_{\sigma}^{e}-m_{\tau+}^{e}\right)$ for $\sigma, \tau \in \mathcal{T}$ and $\sigma \leq \tau$.

Example 3. We return to the setting of Example 1. Fix $t<1, F \in \mathcal{F}_{t}$ and let $f \in L^{\infty}\left(\Omega, \mathcal{F}_{1}, P\right)$. Then by Fatou's lemma and ordinary properties of the Banach limit

$$
P\left(\varlimsup_{n} \frac{P\left(f Z_{1-2^{-n}} \mid \mathcal{F}_{t}\right)}{Z_{t}} Z_{t} F\right) \geq \mu(f F) \geq P\left(\underline{\lim }_{n} \frac{P\left(f Z_{1-2^{-n}} \mid \mathcal{F}_{t}\right)}{Z_{t}} Z_{t} F\right)
$$

(4.8) illustrates that the difficulty of proving the existence of the conditional expectation of $\mu$ with respect to $\mathcal{F}_{t}$ lies in the fact that the sequence $\left\langle P\left(f Z_{1-2^{-n}} \mid \mathcal{F}_{t}\right)\right\rangle_{n \in \mathbb{N}}$ may not converge $P$ a.s. for some $f \in L^{\infty}\left(\Omega, \mathcal{F}_{1}, P\right)$ and $t \in \mathbb{R}_{+}$. More precisely,

Lemma 3. Let $Z=\left(Z_{t}: t \in \mathbb{R}_{+}\right)$be a positive martingale. Then $Z$ is uniformly integrable if and only if the sequence $\left\langle P\left(f Z_{1-2^{-n}} \mid \mathcal{F}_{t}\right)\right\rangle_{n \in \mathbb{N}}$ converges $P$ a.s. for all $f \in L^{\infty}\left(\Omega, \mathcal{F}_{1}, P\right)$ and some $t \in \mathbb{R}_{+}$.

Proof. If $Z$ is uniformly integrable, then $Z_{1-2^{-n}}=P\left(Z \mid \mathcal{F}_{1-2^{-n}}\right)$ for some $Z \in L^{1}(\Omega, \mathcal{F}, P)_{+}$so that $P\left(Z_{1-2^{-n}} f \mid \mathcal{F}_{t}\right)=P\left(Z P\left(f \mid \mathcal{F}_{1-2^{-n}}\right) \mid \mathcal{F}_{t}\right)$. Then $P\left(f \mid \mathcal{F}_{1-2^{-n}}\right)$ converges $P$ a.s. and the claim follows from Fatou's lemma as applied to conditional expectation. Conversely, if $\left\langle P\left(f Z_{1-2^{-n}} \mid \mathcal{F}_{t}\right)\right\rangle_{n \in \mathbb{N}}$ converges $P$ a.s. then, given that $\left|P\left(f Z_{1-2^{-n}} \mid \mathcal{F}_{t}\right)\right| \leq\|f\| Z_{t}$ for $1-2^{-n} \geq t$, by Lebesgue dominated convergence $\lim _{n} P\left(f Z_{1-2^{-n}}\right)$ exists for all $f \in L^{\infty}\left(\Omega, \mathcal{F}_{1}, P\right)$ so that the martingale $\left(Z_{1-2^{-n}}: n \in \mathbb{N}\right)$ is uniformly integrable so that for $1-2^{-n}>t, Z_{t}=P\left(Z_{1-2^{-n}} \mid \mathcal{F}_{t}\right)=P\left(P\left(Z_{\infty} \mid \mathcal{F}_{1-2^{-n}}\right) \mid \mathcal{F}_{t}\right)=P\left(Z_{\infty} \mid \mathcal{F}_{t}\right)$.

As $\mu$ is purely finitely additive $\left\langle P\left(f Z_{1-2^{-n}} \mid \mathcal{F}_{t}\right)\right\rangle_{n \in \mathbb{N}}$ will not converge $P$ a.s. for some $f \in L^{\infty}\left(\Omega, \mathcal{F}_{1}, P\right)$ and all $t \in \mathbb{R}_{t}$. Martingale convergence provides a way out. Choose

$$
\eta_{f, F}=\frac{\mu(f F)-P\left(\underline{\lim }_{n} P\left(f Z_{1-2^{-n}} \mid \mathcal{F}_{t}\right) F\right)}{P\left(\varlimsup_{n} P\left(f Z_{1-2^{-n}} \mid \mathcal{F}_{t}\right) F\right)-P\left(\underline{\lim }_{n} P\left(f Z_{1-2^{-n}} \mid \mathcal{F}_{t}\right) F\right)}
$$

and set

$$
\mu(f \mid F)=\eta_{f, F} \varlimsup_{n} \frac{P\left(f Z_{1-2^{-n}} \mid \mathcal{F}_{t}\right)}{Z_{t}}+\left(1-\eta_{f, F}\right) \underline{\lim }_{n} \frac{P\left(f Z_{1-2^{-n}} \mid \mathcal{F}_{t}\right)}{Z_{t}}
$$

Then $\mu(\mu(f \mid F) F)=P\left(\mu(f \mid F) F Z_{t}\right)=\mu(f F)$. Let $\Pi$ be the collection of all finite, $\mathcal{F}_{t}$ measurable partitions of $\Omega$. For $\pi \in \Pi$ define $\mu(f \mid \pi)=\sum_{F \in \pi} \mu(f \mid F) F$ and let $\mathcal{F}_{\pi}$ be the $\sigma$ algebra generated by $\pi$. 
Thus $(\mu(f \mid \pi): \pi \in \Pi)$ is a uniformly integrable martingale on the filtration $\left(\mathcal{F}_{\pi}: \pi \in \Pi\right)$ and therefore it converges in $L^{1}(P)$ (but not necessarily $P$ a.s.) to a limit, $\mu\left(f \mid \mathcal{F}_{t}\right)$. We thus conclude that

$$
\mu(f F)=\lim _{\pi} P\left(\mu(f \mid \pi) F Z_{t}\right)=P\left(\mu\left(f \mid \mathcal{F}_{t}\right) F Z_{t}\right)=\mu\left(\mu\left(f \mid \mathcal{F}_{t}\right) F\right)
$$

4.3. An Explicit Representation. In this section we shall construct an explicit representation for the expectation $m(k)$ whenever $k \in \mathcal{K}$ and $m \in \mathcal{M}(\mathcal{C})$.

Let $P \in \mathbb{P}(m)$ be fixed throughout this section and $M-A$ be the Doob Meyer decomposition of $X^{6}$ as the difference of a local martingale and a predictable, increasing process and denote by $\mathcal{T}_{X}$ the collection of stopping times $\tau$ such that $X^{\tau}$ is of class $D$. Let $\mathbb{D}$ represent the class of all processes adapted to the filtration and with càdlàg paths save possibly on a set of $P$ measure zero. By $\mathfrak{D}$ we shall indicate the collection of all finite subsets of $\mathbb{D}$ containing the deterministic time process and $M^{*}-$ where $M_{t}^{*}=\sup _{s \leq t} M_{s}$. For each $d \in \mathfrak{D}$ let $\eta_{d}=1 / \# d$ and, notwithstanding the potential incompleteness of the filtration - see [27, lemma I.1.19, p. 5] - define the following sequence $\left\langle t_{i}^{d}\right\rangle_{i \in \mathbb{N}}$ of stopping times : $t_{0}^{d}=0$

$$
t_{i}^{d}=\inf \left\{t>t_{i-1}^{d}: \bigvee_{X \in d}\left|X_{t}-X_{t_{i-1}^{d}}\right| \geq \eta_{d}\right\}
$$

By definition, $t_{i+1}^{d} \leq t_{i}^{d}+\eta_{d} \leq(i+1) \eta_{d}$ and $M_{t}^{t_{i}^{d}}=M_{t}\left\{t<t_{i}^{d}\right\}+M_{t_{i}^{d}}\left\{t \geq t_{i}^{d}\right\} \leq \eta_{d}+M_{t_{i-1}^{d}}+M_{t_{i}^{d}}$ so that $t_{i}^{d} \in \mathcal{T}_{X} i \geq 1$. Since $\left\langle t_{i}^{d}\right\rangle_{i \in \mathbb{N}}$ increases to $\infty P$ a.s., we can choose an integer $I_{d}$ sufficiently large so that $P\left(t_{I_{d}}^{d}<2^{1 / \eta_{d}}\right)<\eta_{d}$. Consider the following quantities

$$
J_{d}^{p}(K)=\sum_{i=0}^{I_{d}-1} m_{t_{i+1}^{d}}^{p}\left(K_{t_{i+1}^{d}}-K_{t_{i}^{d}}\right) \text { and } J_{d}^{e}(K)=\sum_{i=0}^{I_{d}-1} m_{t_{i+1}^{d}}^{e}\left(K_{t_{i+1}^{d}}-K_{t_{i}^{d}}\right)
$$

so that

$$
m\left(K_{t_{I_{d}}^{d}}\right)=\sum_{i=0}^{I_{d}-1} m\left(K_{t_{i+1}^{d}}-K_{t_{i}^{d}}\right)=\sum_{i=0}^{I_{d}-1}\left(m_{t_{i+1}^{d}+}^{e}+m_{t_{i+1}^{d}+}^{p}\right)\left(K_{t_{i+1}^{d}}-K_{t_{i}^{d}}\right)=J_{d}^{p}(K)+J_{d}^{e}(K)
$$

Exploiting Theorem 5(ii) and (4.9) we obtain

$$
\begin{aligned}
J_{d}^{e}(K) & =P \sum_{i=0}^{I_{d}-1} X_{t_{i+1}^{d}}\left(K_{t_{i+1}^{d}}-K_{t_{i}^{d}}\right) \\
& =P\left\{\sum_{i=0}^{I_{d}-1}\left(X_{t_{i+1}^{d}} K_{t_{i+1}^{d}}-X_{t_{i}^{d}} K_{t_{i}^{d}}\right)+\sum_{i=0}^{I_{d}-1}\left(X_{t_{i}^{d}}-X_{t_{i+1}^{d}}\right) K_{t_{i}^{d}}\right\} \\
& =P\left\{X_{t_{I_{d}}^{d}} K_{t_{I_{d}}^{d}}+\sum_{i=0}^{I_{d}-1}\left(A_{t_{i+1}^{d}}-A_{t_{i}^{d}}\right) K_{t_{i}^{d}}\right\}
\end{aligned}
$$

On the other hand, Lemma 6 in the Appendix delivers

$$
J_{d}^{p}(K)=P \sum_{i=0}^{I_{d}-1}\left(m_{t_{i+1}^{d}+}^{p}\left(K_{t_{i+1}^{d}} \mid \mathcal{I}_{t_{i}^{d}+}\right)-K_{t_{i}^{d}}\right)\left(A_{t_{i+1}^{d}}-A_{t_{i}^{d}}\right)
$$

where $m_{t_{i+1}^{d}+}^{p}\left(K_{t_{i+1}^{d}} \mid \mathcal{I}_{t_{i}^{d}+}\right)$ is defined as in Corollary 2. We conclude

$$
m\left(K_{t_{I_{d}}^{d}}\right)=P\left\{X_{t_{I_{d}}^{d}} K_{t_{I_{d}}^{d}}+\int \mathcal{P}_{d}(K) d A\right\}
$$

\footnotetext{
${ }^{6}$ As defined in Theorem $5(i i)$ and avoiding reference to $P$.
} 
where we have implicitly defined

$$
\left.\left.\left.\left.\mathcal{P}_{d}(f)=\sum_{i=0}^{I_{d}-1} \bar{m}_{t_{i+1}^{d}+}^{p}\left(f_{t_{i+1}^{d}} \mid \mathcal{I}_{t_{i}^{d}+}\right)\right]\right] t_{i}^{d}, t_{i+1}^{d}\right]\right], \quad f \in \mathfrak{B}(\tilde{\mathcal{F}})
$$

with $\bar{m}_{t_{i+1}^{d}+}^{p}$ a fixed, positive extension of $m_{t_{i+1}^{d}+}^{p}$ to $\mathcal{F}$. Define also $\lambda \in c a(\mathcal{P})_{+}$implicitly by letting $\lambda(F)=P \int F d A$ for each $F \in \mathcal{P}$.

On these simple remarks is based the proof of the following

Proposition 2. Let $\tau \in \mathcal{T}_{X}, K \in \mathbb{K}$ and assume that the market is free of arbitrage opportunities. Then

$$
0=P\left\{X_{\tau} K_{\tau}+\int_{0}^{\tau} \mathcal{P}_{m}(K) d A\right\}
$$

where for each $f \in \mathfrak{B}(\tilde{\mathcal{F}}), \mathcal{P}_{m}(f) \in L^{\infty}(\lambda)$ is the unique solution to the equation

$$
\underset{d}{\operatorname{LIM}} P \int h \mathcal{P}_{d}(f) d A=P \int h \mathcal{P}_{m}(f) d A, \quad h \in L^{1}(\lambda)
$$

Moreover:

(a). The operator $\mathcal{P}_{m}: \mathfrak{B}(\tilde{\mathcal{F}}) \rightarrow L^{\infty}(\lambda)$ is positive, linear and $\left\|\mathcal{P}_{m}\right\|=1$;

(b). $\mathcal{P}_{m}(f h)=\mathcal{P}_{m}(f) h$ whenever $f, h \in \mathfrak{B}(\tilde{\mathcal{F}})$ and $h$ is càglàd.

With an eye to the financial implications of Proposition 2 (to be developed in the next section) we introduce the notion of covariation between a measure and a semimartingale, a rather natural generalization of the familiar concept of covariation between two semimartingales. A fairly intuitive starting point is the quantity

$$
\sum_{i=0}^{I_{d}-1}\left(\bar{m}_{t_{i+1}^{d}}-\bar{m}_{t_{i}^{d}}\right)\left(f_{t_{i+1}^{d}}-f_{t_{i}^{d}}\right), \quad f \in \mathfrak{B}(\tilde{\mathcal{F}})
$$

where $\bar{m}_{t_{i}^{d}}=\bar{m}_{t_{i}^{d}+}^{e}+\bar{m}_{t_{i}^{d}+}^{p}$ and $\bar{m}_{t_{i}^{d}+}^{e}$ and $\bar{m}_{t_{i}^{d}+}^{p}$ are positive extensions of $m_{t_{i}^{d}+}^{e}$ and $m_{t_{i}^{d}+}^{p}$ to $\mathcal{F}$ respectively with $\bar{m}_{t_{i}^{d}+}^{p}$ arbitrary and $\bar{m}_{t_{i}^{d}+}^{e}$ defined so as to admit $X_{t_{i}^{d}}$ as $P$ density. For every bounded $P$ semimartingale $f$ we define

$$
\left(m^{p}, f\right)=\int\left[\mathcal{P}_{m}(f)-f\right] d A \quad \text { and } \quad[m, f]=[M, f]+\left(m^{p}, f\right)
$$

The notational choice adopted may be justified on the ground of the following characterization

Theorem 6. For each $d \in \mathfrak{D}$ and $0 \leq i<I_{d}$, let $\bar{m}_{t_{i}^{d}}$ be defined as in the text. Let $f$ be a bounded $P$ semimartingale and fix $\tau \in \mathcal{T}$ such that $M_{-}^{\tau}$ is bounded and that $f$ decomposes into the sum of a square integrable martingale and a process of integrable variation. Then the process $[m, f]$ defined in (4.15) is such that

$$
P\left([m, f]_{\tau}\right)=\operatorname{LIM}_{d} \sum_{i=0}^{I_{d}-1}\left(\bar{m}_{t_{i+1}^{d}}-\bar{m}_{t_{i}^{d}}\right)\left(f_{t_{i+1}^{d}}^{\tau}-f_{t_{i}^{d}}^{\tau}\right)
$$

If $f=h . g$ with $g$ and $h$ bounded and $h$ càglàd then $[m, f]=\int h d[m, g]$. 


\section{The Martingale Property}

In this section we take as given $m \in \mathcal{M}(C), P \in \mathbb{P}(m)$ and $X=M-A-$ as defined in Theorem 5 . Denote by $\mathcal{P}(\cdot)$ the $P$ predictable projection and by $(\cdot)^{\mathcal{P}}$ the $P$ predictable compensator of a finite variation process. We introduce the stopping times

$$
T_{n}=\inf \left\{t \in \mathbb{R}_{+}: X_{t} \leq n^{-1}\right\} \quad \text { and } \quad T=\inf \left\{t \in \mathbb{R}_{+}: X_{t-}=0 \text { or } X_{t}=0\right\}
$$

A first implication of Proposition 2 is the semimartingale property of asset returns with respect to $P$.

Theorem 7. Let $K \in \mathbb{K}$. In the absence of arbitrage opportunities the process $K^{T}$ is a $P$ semimartingale. Moreover, if financial markets are complete over finite horizons and $\mathbb{P}(\mathcal{F}, \mathcal{N}) \neq \varnothing$, then $P$ may be chosen such that $P(T<\infty)=0$, so that $K$ is a $P$ semimartingale.

Proof. By localization, we can assume temporarily that (4.13) holds for every $\tau \in \mathcal{T}$. Observe that the process $Y_{t}=X_{t} K_{t}+\int_{0}^{t} \mathcal{P}_{m}(K) d A$ is right continuous, admits a terminal variable and $Y_{0}=0$. Then, [27, lemma I.1.44], $Y$ is a uniformly integrable martingale, i.e. $X_{t} K_{t}=Y_{t}-\int_{0}^{t} \mathcal{P}_{m}(K) d A$ a special semimartingale, given that $\int \mathcal{P}_{m}(K) d A$ is predictable. If $W$ is a bounded process, then $W^{T}[[T, \infty[[$ consists of a bounded jump at time $T$ and is therefore càdlàg and of integrable variation, i.e. a semimartingale. It follows that $K^{T}[[T, \infty[[$ and $D=[[T, \infty[[$ are semimartingales as well as the process

$$
X K+K^{T}\left[\left[T, \infty\left[\left[=(X+D) K^{T}=U K^{T}\right.\right.\right.\right.
$$

$U$ is a strictly positive semimartingale, as $P\left(X_{t}=0 ; t<T\right)=0$. Let

$$
R_{n}=\inf \left\{t \in \mathbb{R}_{+}: \sup _{s \leq t} X_{s}>2^{n} \text { or } X_{t} \leq 2^{-n}\right\}
$$

and let superscript $n$ denote a process stopped before time $R_{n}$, i.e. $U^{n}=U^{R_{n}-}$. $U^{n}$ takes its values in the compact set $\left[2^{-n}, 2^{n}\right]$ on which the inverse function $h$ is well defined and, being convex, admits a Lipschitz constant $c_{n}$. Let $F \in \mathcal{F}_{s}$ and $s<t$. Then $\left|h\left(X_{t}^{n}+D_{t}^{n}\right)-h\left(X_{t}^{n}+D_{s}^{n}\right)\right| \leq c_{n}\left(D_{t}^{n}-D_{s}^{n}\right)$ so that

$$
\begin{aligned}
P\left(h\left(X_{t}^{n}+D_{t}^{n}\right)+c_{n}\left(D_{t}^{n}-D_{s}^{n}\right) \mid \mathcal{F}_{s}\right) & \geq P\left(h\left(X_{t}^{n}+D_{s}^{n}\right) \mid \mathcal{F}_{s}\right) \\
& \geq h\left(P\left(X_{t}^{n}+D_{s}^{n} \mid \mathcal{F}_{s}\right)\right) \\
& \geq h\left(X_{s}^{n}+D_{s}^{n}\right)
\end{aligned}
$$

In other words, $h\left(U^{n}\right)+c_{n} D^{n}$ is a submartingale therefore $h\left(U^{n}\right)=h(U)^{R_{n}-}$ is a semimartingale. As the sequence $\left\langle R_{n}\right\rangle_{n \in \mathbb{N}}$ increases to $\infty, P$ a.s. it follows [34, theorem 6, p. 54] that $h(U)=U^{-1}$ is a semimartingale. But then $K^{T}$, being the product of two semimartingales, is itself a semimartingale by Ito's lemma.

If $F \in \mathcal{I}_{t+\epsilon}$ then

$$
m(F ; T \leq t)=m_{t+\epsilon}^{e}(F ; T \leq t) \leq m_{t+}^{e}(F ; T \leq t) \leq P\left(X_{t}\{T \leq t\}\right)=0
$$

so that if markets are complete $\{F ; T \leq t\} \in \mathcal{N}$, by Theorem 3. But under the current assumptions and by Theorem 5, we conclude that $P$ vanishes on $\mathcal{N}$. Then, $P(F ; T \leq t)=0$ for each $F \in \mathcal{I}_{t}$ i.e. $P(T \leq t)=0$ by Lemma $2(i i)$ and the claim follows.

The absence of arbitrage opportunities is then enough to imply the semimartingale nature of asset returns, a pervasive assumption in all financial models. It should be highlighted that, as is well known, there are 
predecessors to this result, particularly Ansel and Stricker [3, theorem 8, p. 383] and Stricker [40, theorem 3 p. 456 and theorem 5, p. 458] (but see also [16, theorem 7.2, p. 504]). The noticeable fact is that this property, which crucially depends on the underlying probability measure, is obtained here with no preassigned probability: it is therefore entirely endogenous. Of course, the behavior of $K$ after the random time $T$ is totally unrestricted: in fact our model contains no prediction over $]] T, \infty[[$. The issue of whether $P(T<\infty)>0$ will be treated in some detail in the next section and for this reason it will not be discussed further here, save to remark that market completeness is a sufficient condition for $T=\infty$ : once again the implicit probabilistic model turns out to depend in a crucial way on the structure of markets.

For each $K \in \mathbb{K}$ let $M^{K}+V^{K}$ be the canonical decomposition of $\left\{X_{-}>0\right\} . K$, with $M^{K}$ a local martingale and $V^{K}$ predictable and of locally integrable variation. Theorem 7 makes it possible to fully develop the financial implications of (4.13) via integration by parts formula. In particular, letting superscript $\mathcal{P}$ denote the predictable compensator (relative to $P$ ), we have the canonical decomposition

$$
X K+\mathcal{P}_{m}(K) \cdot A=\left(X_{-} . M^{K}+K_{-} \cdot M+[m, K]-[m, K]^{\mathcal{P}}\right)+X_{-} . V^{K}+[m, K]^{\mathcal{P}}
$$

where $X_{-} . M^{K}+\left(X-\mathcal{P}_{P}(X)\right) \cdot V^{K}+K_{-} . M-\left[A, M^{K}\right]$ is a local martingale and $X_{-} . V^{K}+\left[m^{p}, K\right]^{\mathcal{P}}$ a predictable process of finite variation. Denote by $\mathcal{E}$ the exponential semimartingale of Doléans-Dade (and $\mathcal{L}$ its inverse, the stochastic logarithm) and define the positive, right continuous supermartingale $Z=$ $\left(Z_{t}: t \in \mathbb{R}_{+}\right)$implicitly via

$$
Z_{t}=\lim _{n} \mathcal{E}\left(\int_{0}^{t \wedge T_{n}} X_{-}^{-1} d M\right)
$$

where $T_{n}$ is defined as in (5.1) (see Lemma 7 in the Appendix). We obtain the following (where superscript $c$ and $j$ denote the continuous and pure jump parts of a local martingale):

Theorem 8. Let $K \in \mathbb{K}$ and denote by $D_{K}$ the predictable support of the random set $\{\Delta K \neq 0\}$. In the absence of arbitrage opportunities each one the following equivalent conditions holds:

(i). $X_{-} . V^{K}+[m, K]$ is a local martingale so that

$$
V^{K}+\int X_{-}^{-1} d[m, K]^{\mathcal{P}}=0
$$

(ii). on each $\left[\left[0, T_{n}\right]\right], n \in \mathbb{N}$,

$$
V^{K}+[\mathcal{L}(Z), K]+\int X_{-}^{-1} d\left(m^{p}, K\right)^{\mathcal{P}}=0
$$

i.e.

$$
\begin{gathered}
V^{K, j}+\sum \mathcal{P}\left(\Delta \mathcal{L}(Z) \Delta M^{K}\right)+\sum X_{-}^{-1} \mathcal{P}\left(\Delta\left(m^{p}, K\right)\right)=0 \\
V^{K, c}+\left\langle\mathcal{L}(Z), M^{K}\right\rangle+\int X_{-}^{-1} d\left(m^{p}, D_{K}^{c} \cdot K\right)^{c}=0
\end{gathered}
$$

(iii). the process $Z\left(K+X_{-}^{-1} \cdot\left(m^{p}, K\right)^{\mathcal{P}}\right)$ stopped at $T_{n}$ is a local martingale for each $n$.

Proof. As remarked in the proof of Theorem 7, the process $X K+\mathcal{P}_{m}(K) . A$ is a local martingale: (5.2) implies then that $X_{-} . V^{K}+[m, K]$ is a local martingale of locally integrable variation i.e. that $X_{-} . V^{K}+$ $[m, K]^{\mathcal{P}}=0$. (5.4) then follows from $X_{-}^{-1}$ being integrable with respect to $[m, K]^{\mathcal{P}}$. Restricting to $\left[\left[0, T_{n}\right]\right]$ (so that $\mathcal{L}(Z)$ is well defined), (5.5) is immediate given (4.15) and (5.4), (5.6) follows from the fact that local martingales of finite variation are purely discontinuous as well as $V^{K, c}+\left\langle\mathcal{L}(Z), M^{K}\right\rangle+X_{-}^{-1} \cdot\left(m^{p}, K\right)^{c}=$ 0 . To prove (5.7), let $\left\langle\tau_{n}\right\rangle_{n \in \mathbb{N}}$ be a sequence of predictable times exhausting $D_{K}$ and, for each $n$, let 
$\left\langle\tau_{n}^{p}\right\rangle_{p \in \mathbb{N}}$ be the announcing sequence for $\tau_{n}$. Let $\left.\left.\left.\left.D_{K}^{p}=\bigcup_{n}\right]\right] \tau_{n}^{p}, \tau_{n}\right]\right]$, a bounded, càglàd process, converging monotonically to $D_{K}$. So by Theorem 6

$$
\left(m^{p}, D_{K} \cdot K\right)=\lim _{p}\left(m^{p}, D_{K} D_{K}^{p} \cdot K\right)=\lim _{p} \int D_{K}^{p} d\left(m^{p}, D_{K} \cdot K\right)=\int D_{K} d\left(m^{p}, D_{K} K\right)
$$

i.e. $\left(m^{p}, D_{K} K\right)^{c}=0$. Form integration by parts and standard properties of the square bracket process it is clear that on $\left[\left[0, T_{n}\right]\right]$ the predictable, finite variation part of $Z\left(K+X_{-}^{-1} \cdot\left(m^{p}, K\right)^{\mathcal{P}}\right)$ amounts to $Z_{-} \cdot\left(V^{K}+X_{-}^{-1} \cdot[m, K]^{\mathcal{P}}\right) \cdot($ iii) implies

$$
Z_{-} \cdot\left(V^{K}+X_{-}^{-1} \cdot[m, K]^{\mathcal{P}}\right)=0 \quad \text { on } \quad\left[\left[0, T_{n}\right]\right]
$$

Given Lemma 7 and the convention $0 / 0=0, X_{-} / Z_{-}$is càglàd and bounded so that

$$
X_{-} . V^{K}+[m, K]^{\mathcal{P}}=0 \quad \text { on } \quad\left[\left[0, T_{n}\right]\right]
$$

On $\left\{X_{-}=0\right\}$ we have $M=A$ so that $A$ is constant, by uniqueness of the Doob Meyer decomposition: $X_{-} . V^{K}+[m, K]^{\mathcal{P}}=0$ on $\left.]\right] T, \infty\left[\left[\right.\right.$. To conclude that $X_{-} . V^{K}+[m, K]^{\mathcal{P}}=0$ it is then sufficient to show that $X_{T-} . \Delta V_{T}^{K}+\mathcal{P}\left([m, K]_{T}\right)=0$. Let $T^{*}=T$ on $\left\{X_{T-}=0\right\}$ and $T^{*}=\infty$ on $\left\{X_{T-}>0\right\}$. Since $\left[\left[T^{*}\right]\right]=[[0, T]]\left\{X_{-}=0\right\}$ we conclude that $T^{*}$ is a predictable stopping time so that $\mathcal{P}(\Delta[m, K])_{T^{*}}=$ $P\left(\Delta[m, K]_{T^{*}} \mid \mathcal{F}_{T^{*}-}\right)$ on $\left\{X_{T-}=0\right\}[27$, theorem I.2.28, p. 23]. But then,

$$
\begin{aligned}
P\left(\left|\mathcal{P}(\Delta[m, K])_{T}\right|\left\{X_{T-}=0\right\}\right) & \leq P\left(\mathcal{P}\left|(\Delta[m, K])_{T^{*}}\right|\left\{X_{T-}=0\right\}\right) \\
& =P\left(\left|\Delta[m, K]_{T^{*}}\right|\left\{X_{T-}=0\right\}\right) \\
& \leq P \int\left\{X_{-}=0\right\}|d[m, K]| \\
& =0
\end{aligned}
$$

so that $X_{T-} \Delta V_{T}^{K}+\mathcal{P}(\Delta[m, K])_{T}=0 P$ a.s. on the set $\left\{X_{T-}=0\right\}$. Since $\left\{X_{T-}>0\right\}=\bigcup_{n}\left\{T_{n}=T\right\}$ we conclude that

$$
\left(X_{T-} \Delta V_{T}^{K}+\mathcal{P}(\Delta[m, K])_{T}\right)\left\{X_{T-}>0\right\}=\sum_{n}\left(X_{T_{n}-} \Delta V_{T_{n}}^{K}+\mathcal{P}(\Delta[m, K])_{T_{n}}\right)\left\{T_{n}=T\right\}=0
$$

i.e. $X_{-} . V^{K}+[m, K]^{\mathcal{P}}=0$ so that $X_{-} . V^{K}+[m, K]$ is a local martingale.

Most of the financial implications of our model stem from (5.4), where, as customary, it is convenient to interpret the term $V^{K}$ as the implicit risk premium ${ }^{7}$. In rough terms, Theorem 8 confirms, on the one hand, the general intuition underlying classical results of modern financial theory, such as CAPM or CCAPM, but, on the other hand, it contributes to the view that their traditional formulation may lead to overlook potentially important financial phenomena.

(5.4) supports in fact the basic idea that risk premia arise from the covariation of returns with the pricing kernel, a fact well understood in the traditional setting where, by effect of the Girsanov theorem, an absolutely continuous change of the probability measure modifies the characteristics of returns via the covariation of returns with the intervening density process. It is essentially the same transformation that applies here in the shift from $m$ to $P$, notwithstanding the lack of countable additivity. The nice fact is that the failure of countable additivity finds an explicit representation via the process $\left(m^{p}, K\right)$ which ultimately follows from decomposition (4.1) and, essentially, reflects the change through time of the relative weight of

\footnotetext{
${ }^{7}$ Recall that $K$ denotes discounted returns.
} 
the two components, $m_{t}^{e}$ and $m_{t}^{p}$, of the pricing measure. By the brief discussion following Lemma 2, this twist may be interpreted as an indication of the increasing difficulty of recovering the countable additivity property as information piles up. Such intrinsic dynamic of the components of the pricing measure $m$ may indeed be correlated with returns: the expected value of payments accruing later in time will be computed to a larger extent under the "irregular" part of $m$.

On the other hand, however, it is not correct, given decomposition (4.15), to simply reduce risk premia to the correlation with market risk as identified with the process $\mathcal{L}(Z)$. Even assets which are completely uncorrelated with such process may well exhibit excess returns as large as $X_{-}^{-1} \cdot\left(m^{p}, K\right)^{\mathcal{P}}$. In other words, the classical $C A P M$ simply overlooks the additional term $X_{-}^{-1} \cdot\left(m^{p}, K\right)^{\mathcal{P}}$ which, being specific of finite additivity, may rightly be interpreted as the purely finitely additive premium. Our model, thus, accounts for possible failures of classical theories of financial returns as it allows to recover one additional factor, neglected in classical models. Indeed a remarkable number of contributions to the risk premium literature has tried to surmount the difficulties inherent in the $C A P M$, starting with the well known three-fund lemma of Merton [32]. More recently, the focus has been on variants of the CCAPM based on special characteristics of preferences or beliefs by the effect of which additional factors, further to marginal utility, naturally arise ${ }^{8}$. In comparison with this literature, our model does not restrict preferences in any way save by requiring that elements of $\mathfrak{B}(\mathcal{F}, \mathcal{N})_{++}$be strictly preferred to 0 : the purely finitely additive premium emerges rather as a natural implication of the shift from the pricing to the representing measure.

The last property listed in Theorem 8 provides a look, from the viewpoint adopted here, at another core issue of financial modeling: the existence of a state price density process, a positive martingale $Z$ transforming asset returns into local martingales. This property has its corresponding version in our setting, although turning returns into local martingales is less straightforward than usual and implies augmenting the return process by the term $X_{-}^{-1} \cdot\left(m^{p}, K\right)^{\mathcal{P}}$, i.e. by that part of the risk premium which is left unexplained by the density process. It is also worth noting that in the traditional setting the existence of the local martingale $Z$ does not bear any relationship to arbitrage, save when of class $D$ and strictly positive.

The preceding remarks spur interest for the financial properties of the purely finitely additive premium. To this end the most remarkable fact is that càglàd returns do not imply any such premium, as $\mathcal{P}_{m}(K)=K$ for this class of processes: in other terms $\left(m^{p}, K\right)$ only depends on the jumps of the return process. A first implication is for financial modeling: the extension of asset pricing models to include possible discontinuities may result in a significant innovation with respect to traditional explanations of the equity premium, a finding that contrasts blatantly with other $C A P M$ like models with jumps, e.g. [5] and [28]. A deeper implication concerns the impact on pricing of jumps, i.e. of events which occur relatively rarely. Examples of discontinuities of returns include a number of different phenomena such as corporate actions, rating downgrading, or, on the aggregate level, sudden drops in the market index. Although the process $\left(m^{p}, K\right)$ is an expression of such events, it is not possible to conclude that it is itself a pure jump process. Put it differently, there could be exceptional market events that do wield a long lasting influence on pricing through

\footnotetext{
${ }^{8}$ In a model with habit formation, Detemple and Zapatero [17, equation (6.5), p. 1647] characterize the second factor as covariance with disutility of future standards of living. In the context of stochastic differential utility, Duffie and Epstein [19, equation (18), p. 422] recover two additional factors further to equilibrium consumption, one of which being related to market portfolio. In a model with differential information, Ziegler [45, equation (24), p. 9] obtains factors ensuing from the updating process. It should be stressed that all these papers consider a model of general equilibrium while our analysis has only a partial equilibrium flavour.
} 
the continuous part of the purely finitely additive premium. By (5.7) this may however be the case only for those discontinuities which occur at non predictable times. Dividend payments, profit warnings or mergers, often announced to the market with due notice, affect risk premia only around the dates at which they take place; aggregate events, like macroeconomic shocks, to the opposite, take lace unpredictably and may thus have a much wider impact on excess returns. This conclusion offers some theoretical support to the point made with great emphasis by Rubinstein [36, pp. 774-775] according to which the stock market crash of October 1987 has determined a structural modification of option prices responsible for the later failure of the formula of Black and Scholes.

\section{Consistent Pricing Measures and The Extension Property.}

It is commonly believed that financial markets are incomplete. However, it is as widely shared the view that any contingent claim may be introduced and traded on the market at a fair price. The pricing measure should then not only be considered as a tool to evaluate currently traded assets, as in the preceding sections, but it should also provide reliable indications for the pricing of claims that do not yet exist on the market but that it may sensible to introduce at some later stage.

To develop this intuition with more rigor we define the extension property.

Definition 4. Let $f \in \mathfrak{B}(\mathcal{F}, \mathcal{N}), \pi \in \mathbb{R}$ and $\mathcal{K}(f ; \pi)=\{k+r(f-\pi): k \in \mathcal{K}, r \in \mathbb{R}\}$. Define

$$
\mathcal{A}(f, \mathcal{K})=\left\{\pi \in \mathbb{R}: \mathcal{K}(f ; \pi) \cap \mathfrak{B}(\mathcal{F}, \mathcal{N})_{+}=\{0\}\right\}
$$

If $\mathcal{A}(f, \mathcal{K}) \neq \varnothing$ then $\mathcal{K}$ is said to possess the extension property with respect to $f ;$ if $\mathcal{A}(f, \mathcal{K}) \neq \varnothing$ for every $f \in \mathfrak{B}(\mathcal{F}, \mathcal{N})$ then $\mathcal{K}$ is said to possess the extension property.

It is clear that the extension property represents a reinforcement of the no arbitrage condition (3.1). The extent of such reinforcement is characterized exactly in Theorem 9 which adopts the following terminology: a subset $F$ of the topological dual $X^{*}$ of a Banach space $X$ is said to be norming for $A \subset X$ whenever $\|x\|=\sup _{f \in F} f(x)$ for each $x \in A$. We also note

$$
\mathcal{M}_{0}=\left\{v \in \mathbb{P}_{b a}(\mathcal{F}, \mathcal{N}): v \ll m \text { for some } m \in \mathcal{M}(\mathcal{C})\right\}
$$

Theorem 9. The following properties are mutually equivalent:

(a) $\mathcal{M}_{0}$ is norming for $\mathfrak{B}(\mathcal{F}, \mathcal{N})_{+}$and such that if $v \in \mathcal{M}_{0}$ and $\left\langle h_{n}\right\rangle_{n \in \mathbb{N}}$ is a sequence in $\mathcal{C}$ with $\lim _{n}\left\|h_{n}^{-}\right\|=0$ and then $h_{n}$ converges to 0 in $v$ measure;

(b) for every $k \in \mathcal{K}$ and $f \in \mathfrak{B}(\mathcal{F}, \mathcal{N}), \underline{\alpha}_{\mathcal{K}}(f) \leq \bar{\alpha}_{\mathcal{K}}(f)$ and $\bar{\alpha}_{k}(f)=\underline{\alpha}_{\mathcal{K}}(f)$ if and only if $\underline{\alpha}_{k}(f)=$ $\bar{\alpha}_{\mathcal{K}}(f) ;$

(c) $\mathcal{A}(f, \mathcal{K})=\left[\underline{\alpha}_{\mathcal{K}}(f), \bar{\alpha}_{\mathcal{K}}(f)\right] \neq \varnothing$ for every $f \in \mathfrak{B}(\mathcal{F}, \mathcal{N})$ - so that $\mathcal{K}$ possesses the extension property;

(d) there are no free lunches, i.e. (3.2) holds.

Proof. $(a) \longrightarrow(b)$. Let $f^{\prime} \in \mathfrak{B}(\mathcal{F}, \mathcal{N}), f=f^{\prime}-\bar{\alpha}_{\mathcal{K}}\left(f^{\prime}\right)$ and $\underline{\alpha}_{k_{0}}\left(f^{\prime}\right)=\bar{\alpha}_{\mathcal{K}}\left(f^{\prime}\right)$ for $k_{0} \in \mathcal{K}:$ then $\underline{\alpha}_{k_{0}}(f)=$ $\bar{\alpha}_{\mathcal{K}}(f)=0$ so that $k_{0}+f \geq 0$ u.n. by (3.5). For each $n$ there is $k_{n} \in \mathcal{K}$ such that $2^{-n}>\bar{\alpha}_{k_{n}}(f)$. Letting 
$h_{n}=\left(1+\left\|k_{0}+f\right\|\right)^{-1}\left(k_{0}-k_{n}\right)$ we conclude that $h_{n} \geq-2^{-n}$ u.n. and that $h_{n} \in \mathcal{K}$. Thus up to negligibility

$$
\begin{aligned}
k_{0}+f & =\left[\left(k_{0}-k_{n}\right)+\left(k_{n}+f\right)\right] \wedge\left\|k_{0}+f\right\| \\
& \leq\left[\left(k_{0}-k_{n}\right)+\bar{\alpha}_{k_{n}}(f)\right] \wedge\left\|k_{0}+f\right\| \\
& \leq\left[\left(k_{0}-k_{n}\right) \wedge\left\|k_{0}+f\right\|\right]+\bar{\alpha}_{k_{n}}(f) \\
& \leq\left(1+\left\|k_{0}+f\right\|\right)\left(h_{n} \wedge 1\right)+\bar{\alpha}_{k_{n}}(f)
\end{aligned}
$$

By $(a), \bar{\alpha}_{k_{0}}(f)=\sup _{v \in \mathcal{M}_{0}} v\left(k_{0}+f\right) \leq \lim _{n} \bar{\alpha}_{k_{n}}(f)$, i.e. $\bar{\alpha}_{k_{0}}(f)=0=\underline{\alpha}_{\mathcal{K}}(f)$ : equivalently, $\bar{\alpha}_{k_{0}}\left(f^{\prime}\right)=$ $\underline{\alpha}_{\mathcal{K}}\left(f^{\prime}\right)$. The proof of the case $\bar{\alpha}_{k_{0}}\left(f^{\prime}\right)=\underline{\alpha}_{\mathcal{K}}\left(f^{\prime}\right)$ is obtained likewise.

$(b) \longrightarrow(c)$. Fix $0 \leq \epsilon \leq 1$ and let $\pi_{\epsilon}(f)=\epsilon \underline{\alpha}_{\mathcal{K}}(f)+(1-\epsilon) \bar{\alpha}_{\mathcal{K}}(f)$. It is clear from (3.3)-(3.4) that $r \pi_{\epsilon}(f)=\pi_{\epsilon}(r f)$ when $r \geq 0$ while $r \pi_{\epsilon}(f)=\pi_{1-\epsilon}(r f)$ when $r \leq 0$. Suppose that $y=k+r\left(f-\pi_{\epsilon}(f)\right) \in$ $\mathfrak{B}(\mathcal{F}, \mathcal{N})_{+}$for some $k \in \mathcal{K}$ and $r \in \mathbb{R}$ and let $f^{\prime}=r f$ and $\epsilon^{\prime}=\epsilon$ if $r>0$ or $\epsilon^{\prime}=1-\epsilon$ otherwise. Then $k+f^{\prime} \geq \pi_{\epsilon^{\prime}}\left(f^{\prime}\right)$ u.n. and therefore $\pi_{\epsilon^{\prime}}\left(f^{\prime}\right) \leq \underline{\alpha}_{k}\left(f^{\prime}\right)$ so that $\underline{\alpha}_{k}\left(f^{\prime}\right)=\bar{\alpha}_{\mathcal{K}}\left(f^{\prime}\right)=\underline{\alpha}_{\mathcal{K}}\left(f^{\prime}\right)=\pi_{\epsilon^{\prime}}\left(f^{\prime}\right)$ and, by $(b), \bar{\alpha}_{k}\left(f^{\prime}\right)=\pi_{\epsilon^{\prime}}\left(f^{\prime}\right)$. But then, $y=k+f^{\prime}-\pi_{\epsilon^{\prime}}\left(f^{\prime}\right) \leq 0$ u.n. so that $\left[\underline{\alpha}_{\mathcal{K}}(f), \bar{\alpha}_{\mathcal{K}}(f)\right] \subset \mathcal{A}(f, \mathcal{K})$. For the reverse, it is obvious that if, say, $\pi>\bar{\alpha}_{\mathcal{K}}(f)$ then there exists $k \in \mathcal{K}$ such that $\pi>\bar{\alpha}_{k}(f)$ so that $-k-(f-\pi)=-k-f+\bar{\alpha}_{k}(f)+\left(\pi-\bar{\alpha}_{k}(f)\right) \geq \pi-\bar{\alpha}_{k}(f)$ u.n. so that $\pi \notin \mathcal{A}(f, \mathcal{K})$. The case $\pi<\underline{\alpha}_{\mathcal{K}}(f)$ is treated likewise.

$(c) \longrightarrow(d)$. Let $f \in \mathfrak{B}(\mathcal{F}, \mathcal{N})_{+}$and $\pi \in \mathcal{A}(f ; \mathcal{K})$. If $m \in \mathbb{P}_{b a}(\mathcal{F}, \mathcal{N})$ separates $\mathcal{K}(f ; \pi)$ and $\mathfrak{B}(\mathcal{F}, \mathcal{N})_{++}$ then $\pi=m(f)$ and $m \in \mathcal{M}(\mathcal{C})$; if, in addition, $f \in \overline{\mathcal{C}}$ then $m(f) \leq 0$. But then $f-\pi \notin \mathfrak{B}(\mathcal{F}, \mathcal{N})_{++}$implies $f=0$ u.n..

$(d) \longrightarrow(a)$. Let $\eta, \epsilon>0$ and $f \in \overline{\mathcal{C}}$ be such that $f>-\epsilon$ u.n.. If $v \in \mathcal{M}(\mathcal{C})$ then $0 \geq v(f)$ and from this we easily deduce that $v(f \geq \eta) \leq \frac{\epsilon}{\epsilon+\eta}$. It follows that every sequence $\left\langle f_{n}\right\rangle_{n \in \mathbb{N}}$ in $\overline{\mathcal{C}}$ such that $\left\|f_{n}^{-}\right\|$ converges to 0 will converge to 0 in $v$ measure for each $v \in \mathcal{M}(\mathcal{C})$, a property that clearly extends to $\mathcal{M}_{0}$. If $(d)$ holds and $F \in \mathcal{F}$ is not negligible there exists $v_{F} \in \mathcal{M}(\mathcal{C})$ that separates $\overline{\mathcal{C}}$ and $\{F\}$ i.e. such that $v_{F}(F)>0$. Then $\bar{v}_{F} \in \mathbb{P}_{b a}(\mathcal{F}, \mathcal{N})$ defined by $\bar{v}_{F}(E)=v_{F}(F)^{-1} v_{F}(E F)$ is clearly in $\mathcal{M}_{0}$ and, letting $F=\{h>(1-\eta)\|h\|\}$ for $h \in \mathfrak{B}(\mathcal{F}, \mathcal{N})_{+}, \bar{v}_{F}(h) \geq(1-\eta)\|h\|$. Thus $\mathcal{M}_{0}$ is norm attaining for $\mathfrak{B}(\mathcal{F}, \mathcal{N})_{+}$ and $(a)$ follows.

The equivalence of $(c)$ and $(d)$ established by Theorem 9 helps translating the abstract notion of absence of free lunches into the practical issue of whether markets may or not be extended consistently with the no arbitrage principle. This provides a sound financial interpretation to the mathematical concept of free lunch, at times criticized for not having a clear market interpretation (see the remarks in [11] and [12]). In their seminal paper, Harrison and Kreps [24, theorem 1, pp. 386-7] had already considered the extension property highlighting its relationship with viability, i.e. the suitability of asset prices to support the optimal choice of an agent with regular preferences (see also [29]). It should however be remarked that our construction does not require restricting preferences so as to satisfy convexity or continuity properties. A version of Theorem 9 could easily be proved by imposing the finite horizon restriction.

It should be remarked that Definition 4 only considers extending financial markets to include just one (although arbitrary) additional claim and it will not be sufficient to guarantee that the market could be extended to any arbitrary set of new contracts consistently with the no arbitrage principle. For example, it may not be possible, in general, to embed the given market structure into one in which financial markets are complete without violating the no arbitrage condition. A market that may be fictitiously completed in 
respect of the absence of arbitrage opportunities will be said to possess the completion property, a considerable strengthening of the extension property considered above. More formally,

Definition 5. $\mathcal{K}$ possesses the completion property if it admits no arbitrage opportunities and, for some $m \in \mathcal{M}(\mathcal{C})$ the set $\mathcal{K}(m)=\{f-m(f): f \in \mathfrak{B}(\mathcal{F}, \mathcal{N})\}$ satisfies

$$
\mathcal{K}(m) \cap \mathfrak{B}(\mathcal{F}, \mathcal{N})_{+}=\{0\}
$$

We illustrate Definition 5 with the following

Example 4. Take the case in which $\Omega=\mathbb{R}_{+}, \mathcal{F}_{t}$ contains all the subsets of $[0, t]$ and $\mathcal{N}=\{\varnothing\}$. Then for each $F \subset[0, t]$ finite and $\lambda \in \mathbb{P}_{b a}(\mathcal{F}), \sum_{x \in F} \lambda(x)=\lambda(F) \leq \lambda([0, t])$ so that, by standard results, $\lambda(x)=0$ for all but finitely many $x \in[0, t]$. In other words there is no $\lambda \in \mathbb{P}_{b a}(\mathcal{F})$ such that $\lambda(f)>0$ whenever $f \in \mathfrak{B}\left(\mathcal{F}_{t}, \mathcal{N}\right)_{++}$. By Theorem 3, this implies that the completion property fails even for finite horizons although the extension property may well hold.

In the light of the preceding example it is then noteworthy that, in the traditional setting, the extension and the completion properties coincide.

Theorem 10. Let $Q \in \mathbb{P}(\mathcal{F}, \mathcal{N})$. In the following $(a) \rightarrow(b) \rightarrow(c)$ and, if $\mathcal{N}=\mathcal{N}_{Q}$, then $(c) \rightarrow(d) \rightarrow(a)$.

(a). $\mathcal{K}$ possesses the extension property;

(b). there exists $m \in \mathcal{M}(\mathcal{C})$ such that $f \in \mathfrak{B}(\mathcal{F}, \mathcal{N})_{+}$and $m(f)=0$ imply $Q(f)=0$;

(c). there exists $m \in \mathcal{M}(\mathcal{C})$ such that $Q \ll m^{e}$;

$(d) . \mathcal{K}$ possesses the completion property

Therefore, in the traditional setting the absence of free lunches implies that each $K \in \mathbb{K}$ is a semimartingale with respect to the given probability measure.

Proof. $(a) \rightarrow(b)$ Let

$$
\eta_{Q}^{m}=\sup \left\{Q(f): f \in \mathfrak{B}(\mathcal{F})_{+},\|f\|_{\mathfrak{B}(\mathcal{F})} \leq 1, m(f)=0\right\} \text { and } \eta_{Q}=\inf _{m \in \mathcal{M}(\mathcal{C})} \eta_{Q}^{m}
$$

In search of a contradiction, suppose that $\eta_{Q}>0$. Then by Lemma 8 in the Appendix (with $\mathcal{M}=\mathcal{M}(\mathcal{C})$ ) there exists $f \in \mathfrak{B}(\mathcal{F})_{+}$such that $Q(f)>0$ while $m(f)=0$ for all $m \in \mathcal{M}(\mathcal{C})$ : given that $Q \in \mathbb{P}(\mathcal{F}, \mathcal{N})$ then necessarily $f \in \mathfrak{B}(\mathcal{F}, \mathcal{N})_{++}$contradicting $(a)$. There exists then a sequence $\left\langle m_{n}\right\rangle_{n \in \mathbb{N}}$ in $\mathcal{M}(\mathcal{C})$ such that if $f \in \mathfrak{B}(\mathcal{F})_{+},\|f\| \leq 1$ and $m_{n}(f)=0$ then $Q(f) \leq 2^{-n}$. Let $\bar{m}=\sum_{n} 2^{-n} m_{n} . \bar{m} \in \mathcal{M}(\mathcal{C})$ and if $f \in \mathfrak{B}(\mathcal{F})_{+}$is such that $\bar{m}(f)=0$ : then $m_{n}(f)=0$ so that $Q(f) \leq 2^{-n}$ for each $n$ i.e. $Q(f)=0$. $(b)$ follows easily.

$(b) \rightarrow(c)$ Let $Q_{m}$ be the component of $Q$ orthogonal to $m^{e}$. Since $m^{p}$ is purely finitely additive, then $Q_{m}$ and $m$ are themselves orthogonal: there is then $F \in \mathcal{F}$ such that $Q(F) \geq \frac{1}{2}\left\|Q_{m}\right\|$ while $m(F)=0$, a contradiction of $(b)$ unless $\left\|Q_{m}\right\|=0$.

$(c) \rightarrow(d)$ If $\mathcal{N}=\mathcal{N}_{Q}$ then $Q \in \mathbb{P}(\mathcal{F}, \mathcal{N})$ so that $(a) \rightarrow(b) \rightarrow(c)$. Let $m$ be as in $(c)$. If $f=f^{\prime}-m\left(f^{\prime}\right) \in$ $\mathfrak{B}(\mathcal{F}, \mathcal{N})_{+}$for some $f^{\prime} \in \mathfrak{B}(\mathcal{F}, \mathcal{N})$ then $m(f)=0$ implies that $m^{e}(f>0)=Q(f>0)=0$ i.e. $f=0$ u.n., proving $(d)$. The implication $(d) \rightarrow(a)$ is trivial.

Eventually, if $\mathcal{N}=\mathcal{N}_{Q}$ choose $m$ as in $(c)$ and let $P \in \mathbb{P}(m, \mathcal{N})$. If $T_{m}^{P}$ is the stopping time associated to the pair $(m, P)$ via (5.1), then necessarily $m^{e}\left(T_{m}^{P}<\infty\right)=0$ i.e. $\left\{T_{m}^{P}<\infty\right\} \in \mathcal{N}$ so that $P\left(T_{m}^{P}<\infty\right)=0$ too. $K$ is then a $P$ semimartingale by Theorem 7 and, given that $P \gg m^{e} \gg Q$, a $Q$ semimartingale too. 
In the traditional setting the absence of free lunches is then equivalent to the completion property so that the restriction to simple integrands contained in Assumption 2 turns then out to be of not so overwhelming importance if there are no free lunches. This, of course, is not enough to imply that the no arbitrage restriction holds for general integrands.

Example 5. Return once more to Example 1 with $Z$ defined as in (3.7). As the return process is continuous, there is no purely finitely additive premium: each $m \in \mathcal{M}(\mathcal{C})$ is associated to some positive local martingale $Z^{m}$ such that, letting $T_{n}^{m}=\inf \left\{t: X_{t}^{m}=2^{-n}\right\}, 2 \sqrt{1-t}=\left\langle\mathcal{L}\left(Z^{m, T_{n}^{m}}\right), K\right\rangle$ for each $n \in \mathbb{N}$. Given that the underlying filtration is generated by the process $B$, then necessarily $\mathcal{L}\left(Z^{m, T_{n}^{m}}\right)=\mathcal{L}\left(Z^{T_{n}^{m}}\right)$, i.e. $Z^{m, T^{m}}=$ $Z^{T^{m}}$ by uniqueness of the Doléans-Dade exponential. Letting $X^{m}$ be the supermartingale associated to $m$, we conclude $X^{m} \leq Z^{T^{m}}$ by Lemma 7 so that $X_{\infty}^{m} \leq X_{\infty}^{m}\left\{T^{m}<\infty\right\}+Z_{\infty}=0$. We deduce that each $m \in \mathcal{M}(\mathcal{C})$ is purely finitely additive and, as a consequence of Theorem 10, that the no free lunch property fails. Nevertheless, since the measure $\mu \in \mathcal{M}(\mathcal{C})$ defined in (3.8) is such that $\mu_{t}^{e} \gg P \mid \mathcal{F}_{t}$, then, again by Theorem 10, the no free lunch property holds over finite horizons.

The preceding Theorem 10 suggests a connection between the extension property and the following condition.

Definition 6. Let $m \in \mathcal{M}(\mathcal{C})$. The pair $(m, P)$ with $P \in \mathbb{P}(m)$ is said to be consistent if for any $f \in$ $\mathfrak{B}(\mathcal{F}, \mathcal{N})_{+}, P(f)>0$ implies $m(f)>0 . m$ is said to be consistent if there exists $P \in \mathbb{P}(m)$ such that $(m, P)$ is consistent.

It is implicit in this definition that $(m, P)$ is consistent only if $P \in \mathbb{P}(\mathcal{F}, \mathcal{N})$ so that for the rest of this section we make the following

Assumption 3. $\mathbb{P}(\mathcal{F}, \mathcal{N}) \neq \varnothing .{ }^{9}$.

To understand better the connection between consistency and the extension property, let $P \in \mathbb{P}(m, \mathcal{N})$, $f \in \mathfrak{B}(\mathcal{F}, \mathcal{N})_{+}$and $P(f)>0$. Then $f$ is not negligible and, as such, a potential new financial claim. However, pricing such claim by $m$ would result in a violation of the no arbitrage principle whenever $m(f)=0$. Therefore to the extent that the market possesses the extension property, one should have a special interest for consistent pricing measures. As a special case of the above, let $f=\left\{T_{m}^{P} \leq t\right\}$ where $T_{m}^{P}$ is defined as in (5.1) and suppose that $P\left(T_{m}^{P} \leq t\right)>0$. Then, by Lemma 2, for each $\epsilon$ there exists a set $F_{\epsilon} \in \mathcal{F}_{t+\epsilon}$ such that $F_{\epsilon} \subset\left\{T_{m}^{P} \leq t\right\}, P\left(F_{\epsilon}\right) \geq(1-\epsilon) P\left(T_{m}^{P} \leq t\right)$ and $m_{t+\epsilon}^{p}\left(F_{\epsilon}\right)=0$. But then

$$
m\left(F_{\epsilon}\right)=m_{t+\epsilon}^{e}\left(F_{\epsilon}\right) \leq m_{t+\epsilon}^{e}\left(T_{m}^{P} \leq t\right) \leq m_{t+}^{e}\left(T_{m}^{P} \leq t\right)=P\left(X_{t}^{P}\left\{T_{m}^{P} \leq t\right\}\right)=0
$$

It is then clear that the consistency of $(m, P)$ requires that $P\left(T_{m}^{P}<\infty\right)=0$. More precisely, recalling remark 2 , page 8 .

Lemma 4. Let $m \in \mathcal{M}(\mathcal{C}), P \in \mathbb{P}(m, \mathcal{N})$ and let $T_{m}^{P}$ be defined as in (5.1). $(m, P)$ is consistent over finite horizons if and only if $P\left(T_{m}^{P}<\infty\right)=0$; it is consistent if and only if $P \ll m^{e}$.

Proof. For $f \in \mathfrak{B}\left(\mathcal{F}_{t}, \mathcal{N}\right)_{+}$

$$
m(f) \geq m_{t+}^{e}(f)=P\left(X_{t}^{P} f\right) \geq P\left(X_{t}^{P} f\left\{T_{m}^{P}(r)>t\right\}\right) \geq 2^{-r} P\left(f\left\{T_{m}^{P}(r)>t\right\}\right)
$$

\footnotetext{
${ }^{9}$ It should be obvious that Assumption 3 is far less restrictive than requiring $\mathcal{N}=\mathcal{N}_{Q}$ for some $Q \in \mathbb{P}(\mathcal{F})$.
} 
where $T_{m}^{P}(r)=\inf \left\{t \in \mathbb{R}_{+}: X_{t} \leq 2^{-r}\right\}$ increases to $T_{m}^{P} P$ a.s.. This and the remarks preceding this Lemma prove the first claim. It is clear that $P \ll m^{e}$ is sufficient for $(m, P)$ to be consistent; it is necessary too as clearly emerges if we choose accurately $F \in \mathcal{F}$ such that $m^{p}(F)=0$ and that the restriction of $P$ to $F$ is orthogonal to $m^{e}$.

In the following Proposition the relationship between the extension property and consistency is spelled out fully.

Proposition 3. Let $Q \in \mathbb{P}(\mathcal{F}, \mathcal{N})$. Then in the absence of free lunches with finite horizon there is $m \in \mathcal{M}(\mathcal{C})$ such that:

(i). for every $P \in \mathbb{P}(m, \mathcal{N})$ the stopping time $T_{m}^{P}$ defined in (5.1) is such that $Q\left(T_{m}^{P}<\infty\right)=0$;

(ii). for every $\epsilon>0$ there exists $P_{\epsilon} \in \mathbb{P}(m, \mathcal{N})$ such that $P_{\epsilon}\left(T_{m}^{P_{\epsilon}}<\infty\right)<\epsilon$.

Proof. Fix $t \in \mathbb{R}_{+}$and suppose that $\eta>0$ is such that for each $m \in \mathcal{M}(\mathcal{C})$ there exists $P \in \mathbb{P}(m, \mathcal{N})$ such that $Q\left(T_{m}^{P} \leq t\right)>\eta$. Then by orthogonality we may find a, $\mathcal{F}_{t}$ measurable subset $F^{m, t} \subset\left\{T_{m}^{P} \leq t\right\}$ such that $Q\left(F^{m, t}\right)>\eta$ and $m\left(F^{m, t}\right)=0$. By Lemma 8, we can find $g_{t} \in \mathfrak{B}\left(\mathcal{F}_{t}\right)_{+}$such that $Q\left(g_{t}\right)>\eta$ and $m\left(g_{t}=0\right)$ for all $m \in \mathcal{M}(\mathcal{C})$. Since $Q \in \mathbb{P}(\mathcal{F}, \mathcal{N})$ this contradicts the absence of free lunches over finite horizon. We can then find a sequence $\left\langle m_{n}\right\rangle_{n \in \mathbb{N}}$ in $\mathcal{M}(\mathcal{C})$ such that $Q\left(T_{m_{n}}^{P_{n}} \leq 2^{n}\right)<2^{-n}$ for any $P_{n} \in \mathbb{P}\left(m_{n}, \mathcal{N}\right)$. Let $m=\sum_{n} 2^{-n} m_{n}$ and $P \in \mathbb{P}(m, \mathcal{N}) \subset \bigcap_{n} \mathbb{P}\left(m_{n}, \mathcal{N}\right)$. Then, $m \in \mathcal{M}(\mathcal{C})$ and $X^{m, P}=\sum_{n} 2^{-n} X^{m_{n}, P}$ so that $\left\{T_{m}^{P} \leq t\right\} \subset \bigcap_{n}\left\{T_{m_{n}}^{P} \leq t\right\}$ for each $t \in \mathbb{R}_{+}$and therefore

$$
Q\left(T_{m}^{P}<\infty\right)=\lim _{n} Q\left(T_{m}^{P}<2^{n}\right) \leq \lim _{n} Q\left(T_{m_{n}}^{P} \leq 2^{n}\right)=0
$$

But then replacing $P$ by $P_{\epsilon}=\epsilon P+(1-\epsilon) Q$ we have that $P_{\epsilon} \in \mathbb{P}(m, \mathcal{N})$ and that $P_{\epsilon}\left(T^{\epsilon}<\infty\right)=$ $\epsilon P\left(T^{\epsilon}<\infty\right)$.

Proposition 3 rules out the case $P\left(T_{m}^{P}=0\right)=1$ in which most of the conclusions of section 5 would actually be vacuous: absence of free lunches with finite horizon is enough to this end. It will however not be true in the general case that the absence of free lunches implies the existence of a consistent pricing measure; neither is this implication holding when the finite horizon requirement is imposed. To this end the existence of a reference probability plays a crucial role. Combining Proposition 3, Theorem 7 and Lemma 7 we easily get

Corollary 1. Assume that $\mathcal{N}=\mathcal{N}_{Q}$ for some $Q \in \mathbb{P}(\mathcal{F})$. Then

(i). the absence of free lunches with finite horizon implies the existence of $m \in \mathcal{M}(\mathcal{C})$ such that $(m, Q)$ is consistent over finite horizons. Therefore each $K \in \mathbb{K}$ is a $Q$ semimartingale and the local martingale $Z$ defined in (5.3) is positive, i.e. $Q\left(Z_{t}=0\right)=0$ for each $t \in \mathbb{R}_{+}$.

(ii). the absence of free lunches implies the existence of $m \in \mathcal{M}(\mathcal{C})$ such that $(m, Q)$ is consistent. Therefore each $K \in \mathbb{K}$ is a $Q$ semimartingale and the local martingale $Z$ defined in (5.3) is strictly positive, i.e. $Q\left(Z_{\infty}=0\right)=0$.

Corollary $1(i)$ is slightly more general than a result of Delbaen and Schachermayer $[16$, theorem 7.2 , p. $504]$. 


\section{Appendix A. Proofs and Additional Results.}

In this appendix we include the proofs omitted in the body of the paper and some auxiliary results.

Proof of Theorem 4. By $(a), f \geq 0$ u.n. if and only if $\{f<0\} \in \mathcal{N}$ and $\|f\|=\left\|f N^{c}\right\|_{\mathfrak{B}(\mathcal{F})}$ for some $N \in \mathcal{N}$. We claim that for each $k \in \mathcal{K}$ there exists $K \in \mathbb{K}$ such that $K_{\infty}=k$ u.n. and $\left\|K^{-}\right\|_{\mathfrak{B}(\tilde{\mathcal{F}})} \leq\left\|k^{-}\right\|:$this is in essence [16, proposition 3.5]. In fact, let $\bar{K} \in \mathbb{K}$ satisfy $\bar{K}_{\infty}=k$ u.n., let $F=\left\{\left\|k^{-}\right\|+\epsilon<-\bar{K}_{t}\right\} \in \mathcal{F}_{t}$ and (recalling, by Theorem 1 , that $k^{-}=\bar{K}_{\infty}^{-}$u.n.) let $N \in \mathcal{N}$ be such that $\left\|\bar{K}_{\infty}^{-} N^{c}\right\|_{\mathfrak{B}(\mathcal{F})}=\left\|k^{-}\right\|$. Then, $\left(\bar{K}_{\infty}-\bar{K}_{t}\right) F N^{c} \in \mathcal{K}$ and $\left(\bar{K}_{\infty}-\bar{K}_{t}\right) N^{c} F \geq-\left(\left\|\bar{K}_{\infty}^{-} N^{c}\right\|_{\mathfrak{B}(\mathcal{F})}+\bar{K}_{t}\right) N^{c} F=-\left(\left\|k^{-}\right\|+\bar{K}_{t}\right) N^{c} F \geq \epsilon N^{c} F$ a contradiction of $(c)$ unless $F \in \mathcal{N}$. By $(a) N_{0}=\bigcup_{q \in \mathbb{Q}_{+}, k \in \mathbb{N}}\left\{\left\|k^{-}\right\|+2^{-k}<-\bar{K}_{q}\right\} \in \mathcal{N}$ so that, replacing $\bar{K}$ by $K=N_{0}^{c} \bar{K}$, we still obtain $K \in \mathbb{K}, k=K_{\infty}$ u.n. and, by right continuity of $\bar{K}$, that $\left\|k^{-}\right\| \geq-K_{t}$ for each $t$, i.e. $\left\|k^{-}\right\| \geq\left\|K^{-}\right\|_{\mathfrak{B}(\tilde{\mathcal{F}})}$.

Let now the sequence $\left\langle f_{n}\right\rangle_{n \in \mathbb{N}}$ be as in $(b)$ and $f$ be its sum. Let $-k_{n} \in \mathcal{K}$ be such that $\bar{\alpha}_{-k_{n}}\left(f_{n}\right) \leq$ $\bar{\alpha}_{\mathcal{K}}\left(f_{n}\right)+\eta 2^{-n}, n \geq 1$. Given (3.5) and $(a)$, we conclude that $f \leq \sum_{n} k_{n}+\sum_{n} \bar{\alpha}_{\mathcal{K}}\left(f_{n}\right)+\eta$ u.n. or even

$$
f \leq\left(\|f\| \wedge \sum_{n} k_{n}\right)+\sum_{n} \bar{\alpha}_{\mathcal{K}}\left(f_{n}\right)+\eta \text { u.n. }
$$

Choose $K^{n} \in \mathbb{K}$ to be such that $K_{\infty}^{n}=k_{n}$ u.n. and $\left\|k_{n}^{-}\right\| \geq\left\|K^{n-}\right\|_{\mathfrak{B}(\tilde{\mathcal{F}})}$. Then since $\sum_{n}\left\|K^{n-}\right\|_{\mathfrak{B}(\tilde{\mathcal{F}})} \leq$ $\sum_{n}\left\|k_{n}^{-}\right\| \leq \sum_{n} \bar{\alpha}_{-k_{n}}\left(f_{n}\right), K=\sum_{n} K_{n} \in \mathbb{K}_{\sigma}($ see $(2.6))$ and $k=\|f\| \wedge \sum_{n} k_{n}=\|f\| \wedge K_{\infty} \in \mathcal{C}_{\sigma}$. By $(c)$ we obtain, as in the proof of Theorem 3 , the existence of $m_{\sigma} \in \mathbb{P}_{b a}(\mathcal{F}, \mathcal{N})$ such that $m_{\sigma}\left[\mathcal{C}_{\sigma}\right] \leq 0$. Consequently $m_{\sigma}(f) \leq m_{\sigma}(k)+\sum_{n} \bar{\alpha}_{\mathcal{K}}\left(f_{n}\right)+\eta \leq \sum_{n} \bar{\alpha}_{\mathcal{K}}\left(f_{n}\right)+\eta$ yielding $m_{\sigma}(f) \leq \sum_{n} \bar{\alpha}_{\mathcal{K}}\left(f_{n}\right)$. Therefore $\lim _{k} m_{\sigma}\left(\sum_{n \geq k} f_{n}\right) \leq \lim _{k} \sum_{n \geq k} \bar{\alpha}_{\mathcal{K}}\left(f_{n}\right)=0$ i.e. $m_{\sigma}(f)=\sum_{n} m_{\sigma}\left(f_{n}\right)$.

Proof of Theorem 5. Let $\tau, v \in \mathcal{T}$ and, for $i \in\{\tau, v\}$, define $\mathcal{G}_{i}=\left\{F\{\tau \leq v\}: F \in \mathcal{F}_{i}\right\}$ and $\phi_{i}=m \mid \mathcal{G}_{i}$. By uniqueness of the decomposition (4.1), $\phi_{i}^{e}=m_{i}^{e} \mid \mathcal{G}_{i}$ and $\phi_{i}^{p}=m_{i}^{p} \mid \mathcal{G}_{i}$. Given that $\mathcal{G}_{\tau} \subset \mathcal{G}_{v}$, we deduce from Lemma $2($ iii $)$ that $\phi_{\tau}^{e} \geq \phi_{v}^{e} \mid \mathcal{G}_{\tau}$ so that

$$
m_{\tau}^{e}(F\{\tau \leq v\}) \geq m_{v}^{e}(F\{\tau \leq v\}), \quad \tau, v \in \mathcal{T}, F \in \mathcal{F}_{\tau}
$$

But then whenever $\tau \in \mathcal{T}, \tau<\infty$ and $F \in \mathcal{F}_{\tau}$,

$$
\begin{aligned}
m_{\tau+}^{e}(F) & =\lim _{n} m_{\tau+2^{-n}}^{e}(F) \\
& =\lim _{n} \sum_{i \geq 0} m_{\tau+2^{-n}}^{e}\left(F\left\{2^{-n} i \leq \tau<2^{-n}(i+1)\right\}\right) \\
& =\lim _{n} \sum_{i \geq 0} m_{\tau+2^{-n}}^{e}\left(F\left\{2^{-n} i \leq \tau<2^{-n}(i+1)\right\}\left\{2^{-n}(i+1) \leq \tau+2^{-n}\right\}\right) \\
& \leq \lim _{n} \sum_{i \geq 0} m_{2^{-n}(i+1)}^{e}\left(F\left\{2^{-n} i \leq \tau<2^{-n}(i+1)\right\}\right)
\end{aligned}
$$

Given that the function $t \rightarrow m_{t}^{e}(\Omega)$ is monotonic, the set $\mathbb{Q}_{+} \cup\left\{t \in \mathbb{R}_{+}: m_{t}^{e}(\Omega)>m_{t+}^{e}(\Omega)\right\}$ is countable. Let $\left\{t_{n}\right\}_{n \in \mathbb{N}}$ be an explicit enumeration and define $\bar{P}=Q+m^{e}+\sum_{n} 2^{-n} \bar{m}_{t_{n}}^{e}$ where $Q \in \mathbb{P}(\mathcal{F})$ is arbitrary and $P=\bar{P}(\Omega)^{-1} \bar{P}$. It is clear that $m_{t_{n}}^{e} \ll P$ for each $n$. However, for $t \in \mathbb{R}_{+}, F \in \mathcal{F}_{t}$ and $n$ such that $t_{n}>t, 0 \leq\left(m_{t}^{e}-m_{t_{n}}^{e}\right)(F) \leq\left(m_{t}^{e}-m_{t_{n}}^{e}\right)(\Omega)$. Hence, given that for each $t \in \mathbb{R}_{+}$and $\epsilon>0$ there exists an integer $n$ such that $t_{n} \geq t$ and $\left(m_{t}^{e}-m_{t_{n}}^{e}\right)(\Omega)<\epsilon, F \in \mathcal{F}_{t}$ and $P(F)=0$ imply $m_{t}^{e}(F)=0 ;$ by $($ A.2) we also conclude that $m_{\tau+}^{e} \ll P \mid \mathcal{F}_{\tau}$ whenever $\tau<\infty$. If $\mathbb{P}(\mathcal{F}, \mathcal{N}) \neq \varnothing$, choosing $Q \in \mathbb{P}(\mathcal{F}, \mathcal{N})$ implies 
$P \in \mathbb{P}(\mathcal{F}, \mathcal{N}) . X^{P}$ is then a $P$ supermartingale by Lemma $2(i i i)$ and is positive and right continuous as $m_{t+}^{e}$ is so setwise.

Proof of Proposition 1. By proving the statement separately for $f^{+}$and $f^{-}$we can reduce to the case where $f \in L^{1}(v)_{+}$. Consider the family $\Pi_{\lambda}$ of all finite, disjoint collections $\pi$ of sets $I \in \mathcal{I}_{\lambda}$ and, for $\pi \in \Pi_{\lambda}$, define $f^{\pi}=\sum_{I \in \pi} \frac{v(f I)}{v(I)} I$. Given that $v(I)=\gamma(I)$ we easily establish that $(i) \gamma\left(f^{\pi}\right)=v\left(f^{\pi}\right)=v\left(f \bigcup_{I \in \pi} I\right) \leq$ $v(f)$, so that $f^{\pi} \in L^{1}(\gamma)_{+}$; besides, $(i i) \gamma\left(f^{\pi} I\right)=v\left(f^{\pi} I\right)=v(f I)$ when $I \in \pi$. Since $f \in L^{1}(v)_{+}$ then for $c>0 v(f>c) \leq c^{-1} v(f)$ so that [20, I.2.20(b), p. 114] for each $\epsilon$ there exists $c^{\epsilon}>0$ such that $v\left(f\left\{f>c^{\epsilon}\right\}\right) \leq \epsilon$. Therefore if $G \in \mathcal{G}$

$$
\begin{aligned}
\gamma\left(f^{\pi} G\right) & =\sum_{I \in \pi} \frac{v(f I)}{v(I)} \gamma(I G) \\
& =v\left(f\left\{f>c^{\epsilon}\right\} \sum_{I \in \pi} I \frac{v(I G)}{v(I)}\right)+\sum_{I \in \pi} \frac{v\left(f I\left\{f \leq c^{\epsilon}\right\}\right)}{v(I)} v(I G) \\
& \leq \epsilon+c^{\epsilon} v(G)
\end{aligned}
$$

It follows that $\gamma\left(f^{\pi}\left\{f^{\pi}>k\right\}\right) \leq \epsilon+c^{\epsilon} v\left(f^{\pi}>k\right) \leq \epsilon+c^{\epsilon} \frac{v\left(f^{\pi}\right)}{k} \leq \epsilon+c^{\epsilon} \frac{v(f)}{k}$ i.e. $\left\{f^{\pi}: \pi \in \Pi_{\lambda}\right\}$ is a weakly compact subset of $L^{1}(\gamma)$. Letting $\Pi_{\lambda}$ be directed by refinement, the net $\left\langle f^{\pi}\right\rangle_{\pi \in \Pi_{\lambda}}$ admits a cluster point $f^{*} \in L^{1}(\gamma)$. Then $\gamma\left(f^{*}\right) \leq v(f)$ by $(i)$ and $v\left(f^{*} I\right)=\gamma\left(f^{*} I\right)=v(f I)$ for each $I \in \mathcal{I}_{\lambda}$, by $(i i)$. As for uniqueness, let $f^{\circ} \in L^{1}(\gamma)$ satisfy (4.6). Since $\mathcal{G}$ is a $\sigma$ algebra and $\gamma$ is countably additive, for any $r$ there exists a set $I_{r} \in \mathcal{I}_{\lambda}$ such that $\gamma\left(I_{r}^{c}\right)<2^{-r}$. Then, $f^{\circ}=f^{*}$ up to a $\gamma$ null set as, for $G \in \mathcal{G}$

$$
\gamma\left(f^{\circ} G\right)=\lim _{r} \gamma\left(f^{\circ} G I_{r}\right)=\lim _{r} v\left(f G I_{r}\right)=\lim _{r} \gamma\left(f^{*} G I_{r}\right)=\gamma\left(f^{*} G\right)
$$

We denote $f^{*}$ by $v\left(f \mid \mathcal{I}_{\lambda}\right)$ : (4.6) follows. Given uniqueness and additivity of $v, v\left(f+g \mid \mathcal{I}_{\lambda}\right)=v\left(f \mid \mathcal{I}_{\lambda}\right)+$ $v\left(g \mid \mathcal{I}_{\lambda}\right) ;(4.7)$ is a consequence of the fact that $I G \in \mathcal{I}_{\lambda}$ whenever $I \in \mathcal{I}_{\lambda}$ and $G \in \mathcal{G}$. For $f \in \mathcal{I}_{\lambda}$ we deduce from (4.7) and (4.6) that $\gamma\left(\left|v\left(f \mid \mathcal{I}_{\lambda}\right)\right|\right)=v(|f|)$ while, in the general case,

$$
\gamma\left(\left|v\left(f \mid \mathcal{I}_{\lambda}\right)\right|\right) \leq \gamma\left(v\left(|f| \mid \mathcal{I}_{\lambda}\right)\right)=\lim _{k} v\left(v\left(|f| \mid \mathcal{I}_{\lambda}\right) I_{k}\right) \leq v(|f|)
$$

It follows that $\left\|v\left(\cdot \mid \mathcal{I}_{\lambda}\right)\right\|=1$.

Let henceforth $m \in \mathcal{M}(\mathcal{C})$ be fixed. For $\tau \in \mathcal{T}$ recall the notation $\mathcal{I}_{\tau}=\mathcal{I}_{m_{\tau}^{p}}$.

Lemma 5. Let $\left\langle\tau_{n}\right\rangle_{n \in \mathbb{N}}$ be a sequence in $\mathcal{T}$ with $\tau_{n} \geq \tau_{n+1}>\lim _{n} \tau_{n}=\tau$. Then,

$$
\lim _{n} m_{\tau_{n}}^{e}(F)=m_{\tau+}^{e}(F), \quad F \in \mathcal{F}_{\tau}
$$

Proof. The inequality $\lim _{n} m_{\tau_{n}}^{e}(F) \leq \sup _{v \in \mathcal{T}, v>\tau} m_{v}^{e}(F)$ is obvious for each $F \in \mathcal{F}_{\tau}$. To prove the converse, let $v \in \mathcal{T}$ be such that $v>\tau$. By (A.1), $m_{\tau_{n}}^{e}\left(F\left\{\tau_{n} \leq v\right\}\right) \geq m_{v}^{e}\left(F\left\{\tau_{n} \geq v\right\}\right)$. But then for $F \in \mathcal{F}_{\tau}$

$$
\lim _{n} m_{\tau_{n}}^{e}(F) \geq \lim _{n} m_{v}^{e}\left(F\left\{\tau_{n} \leq v\right\}\right)=m_{v}^{e}(F)
$$

(A.3) then follows from (4.2).

Corollary 2. Let $f \in L^{1}(m)$. There exists a unique $m\left(f \mid \mathcal{I}_{\tau+}\right) \in L^{1}\left(m_{\tau+}^{e}\right)$ such that

$$
\lim _{n} m\left(f I_{n} b\right)=m_{\tau+}^{e}\left(m\left(f \mid \mathcal{I}_{\tau+}\right) \bigcup_{n} I_{n} b\right), \quad b \in L^{\infty}\left(m_{\tau+}^{e}\right)
$$

for each pair of sequences $\left\langle I_{n}\right\rangle_{n \in \mathbb{N}}$ in $\mathcal{F}$ and $\left\langle\tau_{n}\right\rangle_{n \in \mathbb{N}}$ in $\mathcal{T}$ such that $I_{n} \subset I_{n+1}, \tau_{n} \geq \tau_{n+1}>\lim _{n} \tau_{n}=\tau$ and $I_{n} \in \mathcal{I}_{\tau_{n}}$. The operator $m\left(\cdot \mid \mathcal{I}_{\tau+}\right): L^{1}(m) \rightarrow L^{1}\left(m_{\tau+}^{e}\right)$ is positive, linear and $\left\|m\left(\cdot \mid \mathcal{I}_{\tau+}\right)\right\| \leq 1$. 
Proof. As $f \in L^{1}(m)$ the left hand side of (A.4) is indeed well defined. Consider first the case $f \in \mathfrak{B}(\mathcal{F})$ and $b \in \mathcal{F}_{\tau}$. Let $\left\langle\sigma_{n}\right\rangle_{n \in \mathbb{N}}$ be another sequence in $\mathcal{T}$ decreasing to $\tau$ and such that $\sigma_{n}>\tau$ and let $\left\langle H_{n}\right\rangle_{n \in \mathbb{N}}$ be an increasing sequence with $H_{n} \in \mathcal{I}_{\sigma_{n}}$ and, letting $I=\bigcup_{n} I_{n}$ and $H=\bigcup_{n} H_{n}, m_{\tau+}^{e}(H \Delta I)=0$. Then

$$
\begin{aligned}
\left|m\left(I_{n}-H_{n}\right)\right| & =\left|m_{\tau_{n}}^{e}\left(I_{n}\right)-m_{\sigma_{n}}^{e}\left(H_{n}\right)\right| \\
& \leq\left|m_{\tau_{n}}^{e}(I)-m_{\sigma_{n}}^{e}(H)\right|+m_{\tau_{n}}^{e}\left(I_{n}^{c} I\right)+m_{\sigma_{n}}^{e}\left(H_{n}^{c} H\right)
\end{aligned}
$$

However, $m_{\tau_{n}}^{e}(I)$ and $m_{\sigma_{n}}^{e}(H)$ converge to $m_{\tau+}^{e}(I)$ and $m_{\tau+}^{e}(H)$ respectively by Lemma 5 so that $0=$ $\lim _{n}\left|m_{\tau_{n}}^{e}(I)-m_{\sigma_{n}}^{e}(H)\right|$. On the other hand, letting $N$ be sufficiently large so that $\left\|m_{\tau_{n}}^{e}-m_{\tau+}^{e}\right\|+$ $\left\|m_{\sigma_{n}}^{e}-m_{\tau+}^{e}\right\|<\epsilon / 4$ and for $n>N$,

$$
m_{\tau_{n+p}}^{e}\left(I_{n+p}^{c} I\right)+m_{\sigma_{n+p}}^{e}\left(H_{n+p}^{c} H\right) \leq m_{\tau_{n}}^{e}\left(I_{n+p}^{c} I\right)+m_{\sigma_{n}}^{e}\left(H_{n+p}^{c} H\right)+\epsilon
$$

so that $\lim _{n}\left|m\left(I_{n}-H_{n}\right)\right|=0$. The left hand side of (A.4) is therefore independent of the intervening sequences. In particular let $\left\langle\sigma_{n}\right\rangle_{n \in \mathbb{N}}$ and $\left\langle H_{n}\right\rangle_{n \in \mathbb{N}}$ be sequences as above, $H=\bigcup_{n} H$ and $m_{\tau+}^{e}\left(H^{c}\right)=0$. Then $\lim _{n} m\left(f I_{n} b\right)=\lim _{n} m\left(f H_{n} I b\right)$. Fix then $\left\langle H_{n}\right\rangle_{n \in \mathbb{N}}$ and write $\phi_{\tau}(f ; b)=\lim _{n} m\left(f H_{n} b\right) . \phi_{\tau}(f ; \cdot)$ : $L^{\infty}\left(m_{\tau+}^{e}\right) \rightarrow \mathbb{R}$ is linear and, since $m\left(H_{n}|b|\right) \leq m_{\tau_{n}}^{e}(|b|)$ for each $b \in L^{\infty}\left(m_{\tau+}^{e}\right)$,

$$
\left|\phi_{\tau}(f ; b)\right| \leq\|f\|_{\mathfrak{B}(\mathcal{F})} \lim _{n} m_{\tau_{n}}^{e}(|b|)=\|f\|_{\mathfrak{B}(\mathcal{F})} m_{\tau+}^{e}(|b|)
$$

so that $\phi_{\tau}(f ; \cdot)$ is absolutely continuous with respect to $m_{\tau+}^{e}$. Denote by $m\left(f \mid \mathcal{I}_{\tau+}\right) \in L^{1}\left(m_{\tau+}^{e}\right)$ its Radon Nikodym derivative. Then if the sequence $\left\langle I_{n}\right\rangle_{n \in \mathbb{N}}$ is as in the statement,

$$
\lim _{n} m\left(f I_{n} b\right)=\lim _{n} m\left(f I H_{n} b\right)=\phi_{\tau}(f ; I b)=m_{\tau+}^{e}\left(m\left(f \mid \mathcal{I}_{\tau+}\right) I b\right)
$$

If $f \in L^{1}(m)_{+}$, then define $m\left(f \mid \mathcal{I}_{\tau+}\right)=\lim _{r} m\left(f \wedge r \mid \mathcal{I}_{\tau+}\right), m_{\tau+}^{e}$ a.s.. Indeed, by monotone convergence and given that $m\left((f \wedge r) I_{n}\right)$ converges to $m\left(f I_{n}\right)$ uniformly with respect to $n$ [20, I.7.6, p. 28],

$$
\lim _{n} m\left(f I_{n} b\right)=\lim _{r} \lim _{n} m\left((f \wedge r) I_{n} b\right)=\lim _{r} m_{\tau+}^{e}\left(m\left(f \wedge r \mid \mathcal{I}_{\tau+}\right) I b\right)=m_{\tau+}^{e}\left(m\left(f \mid \mathcal{I}_{\tau+}\right) I b\right)
$$

therefore $m\left(f \mid \mathcal{I}_{\tau+}\right) \in L^{1}\left(m_{\tau+}^{e}\right)_{+}$. If $m_{\tau+}^{e}\left(I^{c}\right)=0$, the inequality

$$
m_{\tau+}^{e}\left(\left|m\left(f \mid \mathcal{I}_{\tau+}\right)\right|\right) \leq m_{\tau+}^{e}\left(m\left(|f| \mid \mathcal{I}_{\tau+}\right)\right) \leq \lim _{n} m\left(|f| I_{n}\right) \leq m(|f|)
$$

implies $\left\|m\left(f \mid \mathcal{I}_{\tau+}\right)\right\| \leq\|f\|$. The general case is treated by considering $f^{+}$and $f^{-}$separately.

Lemma 6. Let $\sigma, \tau \in \mathcal{T}$ with $\sigma<\tau$ and let $K \in \mathbb{K}$. Then

$$
m_{\tau+}^{p}\left(K_{\tau}-K_{\sigma}\right)=\left(m_{\sigma+}^{e}-m_{\tau+}^{e}\right)\left(m_{\tau+}^{p}\left(K_{\tau} \mid \mathcal{I}_{\sigma+}\right)-K_{\sigma}\right)
$$

Proof. Let $F_{n} \in \mathcal{I}_{\left(\sigma+2^{-n}\right) \wedge \tau}$ be such that $P\left(F_{n}\right)>1-2^{-n}$ and fix $I_{r}=\bigcap_{n \geq r} F_{n} \in \mathcal{I}_{\left(\sigma+2^{-r}\right) \wedge \tau}$. Remark that $\left(K_{\tau}-K_{\sigma}\right), I_{r}\left(K_{\tau}-K_{\left(\sigma+2^{-r}\right) \wedge \tau}\right) \in \mathcal{K}$ so that $m\left(K_{\tau}-K_{\sigma}\right)=m\left(I_{r}\left(K_{\tau}-K_{\left(\sigma+2^{-r}\right) \wedge \tau}\right)\right)=0$. Then, by Corollary 2 and (4.3)

$$
\begin{aligned}
m_{\tau+}^{p}\left(K_{\tau}-K_{\sigma}\right) & =-m_{\tau+}^{e}\left(K_{\tau}-K_{\sigma}\right) \\
& =-\lim _{r} m_{\tau+}^{e}\left(I_{r}\left(K_{\tau}-K_{\left(\sigma+2^{-r}\right) \wedge \tau}\right)\right) \\
& =\lim _{r} m_{\tau+}^{p}\left(I_{r}\left(K_{\tau}-K_{\left(\sigma+2^{-r}\right) \wedge \tau}\right)\right) \\
& =\left(m_{\sigma+}^{e}-m_{\tau+}^{e}\right)\left(m_{\tau+}^{p}\left(K_{\tau} \mid \mathcal{I}_{\sigma+}\right)\right)-\lim _{r}\left(m_{\left(\sigma+2^{-r}\right) \wedge \tau}^{e}-m_{\tau+}^{e}\right)\left(I_{r} K_{\left(\sigma+2^{-r}\right) \wedge \tau}\right) \\
& =\left(m_{\sigma+}^{e}-m_{\tau+}^{e}\right)\left(m_{\tau+}^{p}\left(K_{\tau} \mid \mathcal{I}_{\sigma+}\right)-K_{\sigma}\right)
\end{aligned}
$$


Proof of Proposition 2. We adopt here the notation of section 4.3. Let $\mathfrak{D}$ be directed by inclusion and fix $h \in L^{1}(\lambda)$. Set $\Phi(h)=\operatorname{LIM}_{d} P \int \mathcal{P}_{d}(K) h d A$. The inequality

$$
|\Phi(h)| \leq \operatorname{LIM}_{d}\|K\|_{\mathfrak{B}(\tilde{\mathcal{F}})} P \int|h| d A=\|K\|_{\mathfrak{B}(\tilde{\mathcal{F}})}\|h\|_{L^{1}(\lambda)}
$$

establishes that $\Phi$ is a bounded linear operator acting on $L^{1}(\lambda)$ and may thus be represented as an element $\mathcal{P}_{m}(K)$ of $L^{\infty}(\lambda)$ with $\left\|\mathcal{P}_{m}(K)\right\|_{L^{\infty}(\lambda)} \leq\|K\|_{\mathfrak{B}(\tilde{\mathcal{F}})}$. In other words, $\mathcal{P}_{m}: \mathfrak{B}(\tilde{\mathcal{F}}) \rightarrow L^{\infty}(\lambda)$ is a positive, linear operator such that $\left\|\mathcal{P}_{m}\right\| \leq 1$ and solves (4.14).

Let $h \in \mathfrak{B}(\mathcal{P})$ be now càglàd and denote by $h_{+}$its càdlàg counterpart. Then for $d \in \mathfrak{D}$ sufficiently large so that $h_{+} \in d$ we find that for each $i \geq 0$ on the stochastic interval $\left.\left.\left.]\right] t_{i}^{d}, t_{i+1}^{d}\right]\right]$ the inequality $\left|h_{t}-h_{t_{i}^{d}+}\right| \leq \eta_{d}$ holds. But then, given that $\lim _{d} \eta_{d}=0$,

$$
\begin{aligned}
\int \mathcal{P}_{m}(K) h d \lambda & =\operatorname{LIM}_{d} \int \mathcal{P}_{d}(K) h d \lambda \\
& =\operatorname{LIM}_{d} P \sum_{i=0}^{I_{d}-1} m_{t_{i+1}^{d}}^{p}\left(K_{t_{i+1}^{d}} \mid \mathcal{I}_{t_{i}^{d}}^{+}\right) \int_{t_{i}^{d}}^{t_{i+1}^{d}} h d A \\
& =\operatorname{LIM}_{d} P \sum_{i=0}^{I_{d}-1} m_{t_{i+1}^{d}+}^{p}\left(K_{t_{i+1}^{d}} h_{t_{i}^{d}} \mid \mathcal{I}_{t_{i}^{d}}^{+}\right)\left(A_{t_{i+1}^{d}}-A_{t_{i}^{d}}\right) \\
& =\operatorname{LIM}_{d} P \sum_{i=0}^{I_{d}-1} m_{t_{i+1}^{d}+}^{p}\left(K_{t_{i+1}^{d}} h_{t_{i+1}^{d}} \mid \mathcal{I}_{t_{i}^{d}}^{+}\right)\left(A_{t_{i+1}^{d}}-A_{t_{i}^{d}}\right) \\
& =\int \mathcal{P}_{m}(K h) d \lambda
\end{aligned}
$$

which proves claim $(b)$ and entails $\left\|\mathcal{P}_{m}\right\|=1$.

Let eventually $\tau \in \mathcal{T}_{X}$ and $K \in \mathbb{K}$ so that $K^{\tau} \in \mathbb{K}$. Remark that

$$
P\left\{\left\{t_{I_{d}}^{d}<\tau\right\}\left[X_{t_{I_{d}}^{d}} K_{t_{I_{d}}^{d}}^{\tau}+\int \mathcal{P}_{m}\left(K^{\tau}\right) d A\right]\right\} \leq\|K\|_{\mathfrak{B}(\tilde{\mathcal{F}})} P\left(\left\{t_{I_{d}}^{d}<\tau\right\} M_{\tau}\right)
$$

and that

$$
\left.\left.\left.\left.\left.\left.\left.\left.\left.\left.\left.\left.\mathcal{P}_{m}\left(K^{\tau}\right)\right]\right] \tau, t_{I_{d}}^{d}\right]\right]=\mathcal{P}_{m}\left(K_{\tau}\right]\right] \tau, t_{I_{d}}^{d}\right]\right]\right)=K_{\tau}\right]\right] \tau, t_{I_{d}}^{d}\right]\right]
$$

Replacing $K$ by $K^{\tau}$ in (4.11) and taking limits we deduce then

$$
\begin{aligned}
\underset{d}{\operatorname{LIM} m\left(K_{t_{I_{d}}^{d}}^{\tau}\right)} & =\operatorname{LIM}_{d} P\left\{X_{t_{I_{d}}^{d}} K_{t_{I_{d}}^{d}}^{\tau}+\int \mathcal{P}_{m}\left(K^{\tau}\right) d A\right\} \\
& =\operatorname{LIM}_{d} P\left\{\left\{t_{I_{d}}^{d} \geq \tau\right\}\left[X_{t_{I_{d}}^{d}} K_{\tau}+\int_{0}^{\tau} \mathcal{P}_{m}(K) d A+K_{\tau}\left(A_{t_{I_{d}}^{d}}-A_{\tau}\right)\right]\right\} \\
& =P\left\{X_{\tau} K_{\tau}+\int_{0}^{\tau} \mathcal{P}_{m}(K) d A\right\}
\end{aligned}
$$

Proof of Theorem 6. Let us first notice that from (4.15) indeed $[m, f]$ is a process of locally integrable variation for each bounded semimartingale $f$. To prove (4.16), remark that $\bar{m}_{t_{i+1}^{d}+}^{p}-\bar{m}_{t_{i}^{d}+}^{p}$ and $m_{t_{i}^{d}+}^{e}-m_{t_{i+1}^{d}+}^{e}$ coincide in restriction to $\mathcal{F}_{t_{i}^{d}}$, so that the conditional expectation $\left(\bar{m}_{t_{i+1}^{d}+}^{p}-\bar{m}_{t_{i}^{d}+}^{p}\right)\left(\cdot \mid \mathcal{F}_{t_{i}^{d}}\right)$ is well defined, according to Proposition 1 ; moreover, it coincides with $m_{t_{i+1}^{d}}^{p}\left(\cdot \mid \mathcal{I}_{t_{i}^{d}+}\right)^{2+1}$ (and does not depend therefore on 
the choice of the extension $\left.\bar{m}_{t_{i}^{d}+}^{p}\right)$. In fact, for $F \in \mathcal{I}_{t_{i}^{d}}$ and letting $\left\langle I_{n}\right\rangle_{n \in \mathbb{N}}$ be an increasing sequence with $I_{n} \in \mathcal{I}_{\left(t_{i}^{d}+2^{-n}\right) \wedge t_{i+1}^{d}}$ and $P\left(\bigcup_{n} I_{n}\right)=1$ we get

$$
\begin{aligned}
\left(\bar{m}_{t_{i+1}^{d}+}^{p}-\bar{m}_{\left(t_{i}^{d}+2^{-n-p}\right) \wedge t_{i+1}^{d}}^{p}\right)\left(I_{n+p}^{c} I\right)= & \left(m_{\left(t_{i}^{d}+2^{-n-p}\right) \wedge t_{i+1}^{d}}^{e}-m_{t_{i+1}^{d}+}^{e}\right)\left(I_{n+p}^{c} I\right) \\
\leq & \left(m_{\left(t_{i}^{d}+2^{-n}\right) \wedge t_{i+1}^{d}}^{e}-m_{t_{i+1}^{d}}^{e}\right)\left(I_{n+p}^{c} I\right) \\
& +\left\|m_{\left(t_{i}^{d}+2^{-n-p}\right) \wedge t_{i+1}^{d}}^{e}-m_{\left(t_{i}^{d}+2^{-n}\right) \wedge t_{i+1}^{d}}^{e}\right\|
\end{aligned}
$$

so that $\lim _{n}\left(\bar{m}_{t_{i+1}^{d}+}^{p}-\bar{m}_{\left(t_{i}^{d}+2^{-n}\right) \wedge t_{i+1}^{d}}^{p}\right)\left(I_{n}^{c} I\right)=0$ and therefore

$$
\begin{aligned}
P\left\{F m_{t_{i+1}^{d}}^{p}\left(f_{t_{i+1}^{d}} \mid \mathcal{I}_{t_{i}^{d}+}\right)\left(A_{t_{i+1}^{d}}-A_{t_{i}^{d}}\right)\right\} & =\lim _{n} m_{t_{i+1}^{d}+}^{p}\left(f_{t_{i+1}^{d}} I_{n} F\right) \\
& =\lim _{n}\left(\bar{m}_{t_{i+1}^{d}+}^{p}-\bar{m}_{\left(t_{i}^{d}+2^{-n}\right) \wedge t_{i+1}^{d}}^{p}\right)\left(f_{t_{i+1}^{d}} I_{n} F\right) \\
& =\lim _{n}\left(\bar{m}_{t_{i+1}^{d}+}^{p}-\bar{m}_{\left(t_{i}^{d}+2^{-n}\right) \wedge t_{i+1}^{d}}^{p}\right)\left(f_{t_{i+1}^{d}} F\right) \\
& =\lim _{n}\left(\bar{m}_{t_{i+1}^{d}+}^{p}-\bar{m}_{t_{i}^{d}+}^{p}\right)\left(f_{t_{i+1}^{d}} F\right) \\
& =P\left\{\left(\bar{m}_{t_{i+1}^{d}+}^{p}-\bar{m}_{t_{i}^{d}+}^{p}\right)\left(f_{t_{i+1}^{d}} \mid \mathcal{F}_{t_{i}^{d}}\right) F\left(A_{t_{i+1}^{d}}-A_{t_{i}^{d}}\right)\right\}
\end{aligned}
$$

But then

$$
\begin{aligned}
\left(\bar{m}_{t_{i+1}^{d}}-\bar{m}_{t_{i}^{d}}\right)\left(f_{t_{i+1}^{d}}-f_{t_{i}^{d}}\right)= & P\left\{\left(f_{t_{i+1}^{d}}-f_{t_{i}^{d}}\right)\left(X_{t_{i+1}^{d}}-X_{t_{i}^{d}}\right)\right\} \\
& +P\left\{m_{t_{i+1}^{d}}^{p}\left(f_{t_{i+1}^{d}}-f_{t_{i}^{d}} \mid \mathcal{I}_{t_{i}^{d}+}\right)\left(A_{t_{i+1}^{d}}-A_{t_{i}^{d}}\right)\right\}
\end{aligned}
$$

and, by (4.12)

$$
\begin{aligned}
\sum_{i=0}^{I_{d}-1}\left(\bar{m}_{t_{i+1}^{d}}-\bar{m}_{t_{i}^{d}}\right)\left(f_{t_{i+1}^{d}}-f_{t_{i}^{d}}\right)= & P \sum_{i=0}^{I_{d}-1}\left(M_{t_{i+1}^{d}}-M_{t_{i}^{d}}\right)\left(f_{t_{i+1}^{d}}-f_{t_{i}^{d}}\right) \\
& +P \int \mathcal{P}_{d}(f) d A-P \sum_{i=0}^{I_{d}-1} f_{t_{i+1}^{d}}\left(A_{t_{i+1}^{d}}-A_{t_{i}^{d}}\right)
\end{aligned}
$$

Convergence of $P \sum_{i=0}^{I_{d}-1} f_{t_{i+1}^{d}}\left(A_{t_{i+1}^{d}}-A_{t_{i}^{d}}\right)$ to $P \int f d A$ is clear; $\operatorname{LIM}_{d} P \int \mathcal{P}_{d}(f) d A=P \int \mathcal{P}_{m}(f) d A$ was shown in the proof of Proposition 2. (4.16) follows. Let $\tau \in \mathcal{T}_{X}$ so that $M^{\tau}$ is uniformly integrable and assume that $f=f^{\tau}$. Then

$$
P \sum_{i=0}^{I_{d}-1}\left(M_{t_{i+1}^{d}}-M_{t_{i}^{d}}\right)\left(f_{t_{i+1}^{d}}-f_{t_{i}^{d}}\right)=P\left\{M_{t_{I_{d}}^{d}}^{\tau} f_{t_{d}^{d}}-\sum_{i=0}^{I_{d}-1} f_{t_{i}^{d}}\left(M_{t_{i+1}^{d}}-M_{t_{i}^{d}}\right)-\sum_{i=0}^{I_{d}-1} M_{t_{i}^{d}}\left(f_{t_{i+1}^{d}}-f_{t_{i}^{d}}\right)\right\}
$$

so that, given $P \sum_{i=0}^{I_{d}-1} f_{t_{i}^{d}}\left(M_{t_{i+1}^{d}}-M_{t_{i}^{d}}\right)=P \int f_{-} d M=0$ and by bounded convergence for stochastic integrals,

$$
\underset{d}{\operatorname{LIM}} P \sum_{i=0}^{I_{d}-1}\left(M_{t_{i+1}^{d}}-M_{t_{i}^{d}}\right)\left(f_{t_{i+1}^{d}}-f_{t_{i}^{d}}\right)=P\left\{M_{\tau} f_{\tau}-\int_{0}^{\tau} f_{-} d M-\int_{0}^{\tau} M_{-} d f\right\}=P\left([M, f]_{\tau}\right)
$$

If $f=h . g$ with $g$ a semimartingale and $h$ bounded and càglàd, then $[M, f]=\int h d[M, g]$ is clear. On the other hand, it is clear from (4.15) and Proposition 2 that $\left(m^{p}, f\right)=\left(m^{p}, \Delta f\right)$ and that, for $d \in \mathfrak{D}$ sufficiently 
large, $\left|h_{t_{i}^{d}}-h_{t_{i+1}^{d}}\right|<2^{n}$ so that

$$
\begin{aligned}
P \int \mathcal{P}_{m}(\Delta f) d A & =\operatorname{LIM}_{d} \int \mathcal{P}_{d}(\Delta f) d A \\
& =\operatorname{LIM}_{d} P \sum_{i=0}^{I_{d}-1} m_{t_{i+1}^{d}}^{p}\left(h_{t_{i+1}^{d}} \Delta g_{t_{i+1}^{d}} \mid \mathcal{I}_{t_{i}^{d}+}\right)\left(A_{t_{i+1}^{d}}-A_{t_{i}^{d}}\right) \\
& =\operatorname{LIM}_{d} P \sum_{i=0}^{I_{d}-1} m_{t_{i+1}^{d}}^{p}\left(\Delta g_{t_{i+1}^{d}} \mid \mathcal{I}_{t_{i}^{d}+}\right) h_{t_{i}^{d}}\left(A_{t_{i+1}^{d}}-A_{t_{i}^{d}}\right) \\
& =\operatorname{LIM}_{d} P \int \mathcal{P}_{d}(\Delta g) d(h . A) \\
& =\operatorname{LIM}_{d} P \int h \mathcal{P}_{d}(\Delta g) d A \\
& =P \int h \mathcal{P}_{m}(\Delta g) d A
\end{aligned}
$$

so that from $\left(m^{p}, f\right)=\int\left(\mathcal{P}_{m}(\Delta f)-\Delta f\right) d A=\int h\left(\mathcal{P}_{m}(\Delta g)-\Delta g\right) d A=\int h d\left(m^{p}, g\right)$.

Lemma 7. The process $Z$ defined in (5.3) is a positive, right continuous supermartingale such that $Z \geq X$ and that $Z^{T_{n}}$ is a local martingale for each $n \in \mathbb{N}$ - with $T_{n}$ defined as in (5.1).

Proof. That $Z^{T_{n}}$ is a local martingale for each $n$ is pretty clear. $\left(Z_{T_{n}}: n \in \mathbb{N}\right)$ is then a positive supermartingale relative to the filtration $\left(\mathcal{F}_{T_{n}}: n \in \mathbb{N}\right)$ so that $Z_{\infty}=\lim _{n} Z_{n}$ exists then and is finite on the complement of some $P$ null set $E^{c}$ as a consequence of Doob convergence theorem: also, $P\left(\sup _{n} Z_{T_{n}}<\infty\right)=1$. Treating separately the cases $t<T$ and $t \geq T=\lim _{n} T_{n}$ it is clear that $Z_{T_{n} \wedge t}$ converges pointwise on $E: Z_{t}$ then exists for each $t \in \mathbb{R}_{+}-$also $P\left(\sup _{n} Z_{t}^{T_{n}}<\infty\right)=1$ for each $t$. On $\{t \geq T\} Z_{t}=\lim _{n} Z_{T_{n}}$ while for each $\omega \in\{t<T\}=\bigcup_{n}\left\{t<T_{n}\right\}$ there exist $k_{0}>0$ and $n_{0}$ such that $t+2^{-k}<T_{n}$ whenever $k \geq 0$ and $n \geq n_{0}$ so that $Z_{t}(\omega)=Z_{t}^{T_{n}}(\omega)=Z_{t+2^{-k}}^{T_{n}}(\omega)=Z_{t+2^{-k}}(\omega) . Z$ is then right continuous on $E$. Eventually, if $F \in \mathcal{F}_{s}$ and $s<t$

$$
\begin{aligned}
P\left(F Z_{s}\right) & =\lim _{k} \lim _{n} P\left(F Z_{s}^{T_{n}}\left\{\sup _{n} Z_{s}^{T_{n}}<2^{k}\right\}\right) \\
& \geq \lim _{k} \lim _{n} P\left(F Z_{t}^{T_{n}}\left\{\sup _{n} Z_{s}^{T_{n}}<2^{k}\right\}\right) \\
& \geq \lim _{k} P\left(F Z_{t}\left\{\sup _{n} Z_{s}^{T_{n}}<2^{k}\right\}\right) \\
& =P\left(F Z_{t}\right)
\end{aligned}
$$

Moreover

$$
\begin{aligned}
X^{T_{n}} & =\mathcal{E}\left(\int \frac{d M^{T_{n}}}{X_{-}}-\int \frac{d A^{T_{n}}}{X_{-}}\right) \\
& =\exp \left(\int \frac{d M^{T_{n}}}{X_{-}}-\frac{1}{2} \int \frac{d\left\langle M^{T_{n}}\right\rangle}{X_{-}^{2}}-\int \frac{d A^{T_{n}}}{X_{-}}\right) \prod e^{\frac{\Delta X^{T_{n}}}{X_{-}}}\left(1+\frac{\Delta X^{T_{n}}}{X_{-}}\right) \\
& \leq \exp \left(\int \frac{d M^{T_{n}}}{X_{-}}-\frac{1}{2} \int \frac{d\left\langle M^{T_{n}}\right\rangle}{X_{-}^{2}}\right) \prod e^{\frac{\Delta M^{T_{n}}}{X_{-}}}\left(1+\frac{\Delta M^{T_{n}}}{X_{-}}\right) \\
& \leq Z^{T_{n}}
\end{aligned}
$$

But then $X_{t}=X_{t}\{T>t\}=\lim _{n} X_{t}\left\{T_{n}>t\right\}=\lim _{n} X_{t}^{T_{n}}\left\{T_{n}>t\right\} \leq \lim _{n} Z_{t}^{T_{n}}\left\{T_{n}>t\right\} \leq Z_{t}$. 
The key step in the results of section 6 is the following separating lemma, perhaps of its own, independent interest.

Lemma 8. Let $Q \in \mathbb{P}(\mathcal{F})$, let $\mathcal{M}$ be a norm bounded, convex subset of ba $(\mathcal{F})_{+}$and set

$$
\alpha^{*}(f)=\sup _{m \in \mathcal{M}} m(f) \quad f \in \mathfrak{B}(\mathcal{F})
$$

Let $\eta>0$. In the following (a) is equivalent to (b):

(a). for each $m \in \mathcal{M}$ there is $f \in \mathfrak{B}(\mathcal{F})$ with $0 \leq f \leq 1$ satisfying $Q(f) \geq \eta$ and $m(f)=0$;

(b). for each $\epsilon>0$ there is $g \in \mathfrak{B}(\mathcal{F})$ with $0 \leq g \leq 1$ such that $Q(g) \geq \eta(1-\epsilon)$ and $\alpha^{*}(g)=0$.

Proof. $(a) \rightarrow(b)$. For each $m \in \mathcal{M}$ define the set $F_{\epsilon}(m)=\left\{f \in \mathfrak{B}(\mathcal{F})_{+}: f \leq 1, Q(f) \geq \eta(1-\epsilon), m(f)<\epsilon\right\}$ and let $F_{\epsilon}: \mathcal{M} \rightarrow \mathfrak{B}(\mathcal{F})$ be the corresponding set-valued map. $F_{\epsilon}$ is convex valued and, under $(i)$, non empty. Denote by $\mathfrak{X}$ the space $b a(\mathcal{F})$ endowed with the weak* topology (i.e. the topology induced by $\mathfrak{B}(\mathcal{F})$ ), a Hausdorff, locally convex vector space. Consider an open set $\mathcal{U}_{f_{0}} \subset \mathfrak{B}(\mathcal{F})$ containing $f_{0} \in F_{\epsilon}\left(m_{0}\right)$. It is clear that $\mathcal{V}_{f}=\{m \in \mathcal{M}: m(f)<\epsilon\}$ is (relatively) open and that

$$
F_{\epsilon}^{-1}\left(\mathcal{U}_{f_{0}}\right)=\left\{m \in \mathcal{M}: F_{\epsilon}(m) \cap \mathcal{U}_{f_{0}} \neq \varnothing\right\}=\underset{\left\{f \in \mathcal{U}_{f_{0}}: Q(f) \geq \eta(1-\epsilon)\right\}}{\bigcup_{f}} \mathcal{V}_{f}
$$

In other words, the lower inverse $F_{\epsilon}^{-1}$ of $F_{\epsilon}$ maps open sets into open sets, i.e. $F_{\epsilon}$ is lower hemicontinuous. Denote the closure of a set in $\mathfrak{B}(\mathcal{F})$ by an upper bar. By virtue of Michael selection theorem [33, footnote 7, p. 364.], there is a continuous function $\phi_{\epsilon}: \mathfrak{X} \rightarrow \mathfrak{B}(\mathcal{F})$ such that $\phi_{\epsilon}(m) \in \overline{F_{\epsilon}(m)}$ for each $m \in \mathcal{M}$ so that $(i) 0 \leq \phi_{\epsilon}(m) \leq 1,(i i) Q\left(\phi_{\epsilon}(m)\right) \geq \eta(1-\epsilon)$ and $(i i i) m\left(\phi_{\epsilon}(m)\right) \leq \epsilon$.

Consider now the set valued map $M: \mathfrak{B}(\mathcal{F}) \rightarrow \mathfrak{X}$ defined as $M(f)=\left\{m \in \overline{\mathcal{M}}: m(f)=\alpha^{*}(f)\right\} . M(f)$ is clearly a non empty, compact and convex subset of $\mathfrak{X}$. Let $\mathcal{V} \subset \mathfrak{X}$ be closed and such that $M^{-1}(\mathcal{V}) \neq \varnothing$ and choose $f_{0} \in \overline{M^{-1}(\mathcal{V})}$ : for each $\delta$ there exists then $f_{\delta} \in M^{-1}(\mathcal{V})$ such that $\left\|f_{\delta}-f_{0}\right\|<\delta$. By definition this implies that for some $m_{\delta} \in \mathcal{V}, m_{\delta}\left(f_{\delta}\right)=\alpha^{*}\left(f_{\delta}\right)$ so that

$$
m_{\delta}\left(f_{0}\right) \geq m_{\delta}\left(f_{\delta}\right)-\delta=\alpha^{*}\left(f_{\delta}\right)-\delta \geq \alpha^{*}\left(f_{0}\right)-2 \delta
$$

Put it differently, for each $\delta>0$ the set $\mathcal{V}_{f_{0}, \delta}=\left\{m \in \mathcal{V}: m\left(f_{0}\right) \geq \alpha^{*}\left(f_{0}\right)-2 \delta\right\}$ is non empty. It then ensues from the finite intersection property that $\bigcap_{\delta>0} \mathcal{V}_{f_{0}, \delta}=\left\{m \in \mathcal{V}: m\left(f_{0}\right)=\alpha^{*}\left(f_{0}\right)\right\}$ is also non empty or, in other words, that $f_{0} \in M^{-1}(\mathcal{V})$ and therefore $M^{-1}(\mathcal{V})$ is closed. We conclude that $M$ is upper hemicontinuous and that so is the composite map $\Phi_{\epsilon}=M \circ \phi_{\epsilon}: \mathfrak{X} \rightarrow \mathfrak{X}$; further, $\Phi_{\epsilon}$ is convex and compact valued and has therefore closed graph [2, theorem 16.12, p. 529]. But then, as a result of a well known theorem of Glicksberg [23, p. 171] (see also [2, corollary 16.51, p. 550]), $\Phi_{\epsilon}$ admits a fixed point, $m_{\epsilon}$. Letting $f_{\epsilon}=\phi_{\epsilon}\left(m_{\epsilon}\right)$ we have that $\epsilon \geq m_{\epsilon}\left(f_{\epsilon}\right)=\alpha^{*}\left(f_{\epsilon}\right)$ while $Q\left(f_{\epsilon}\right) \geq \eta(1-\epsilon)$. Replacing $\epsilon$ with $\epsilon_{n}=2^{-n}$, let $m_{n}$ be the fixed point of $\Phi_{\epsilon_{n}}$ and $f_{n}=\phi_{\epsilon_{n}}\left(m_{n}\right)$ so that $Q\left(f_{n}\right) \geq \eta\left(1-\epsilon_{n}\right)$ and $\epsilon_{n} \geq \alpha^{*}\left(f_{n}\right)$. Both inequalities remain valid if we replace $f_{n}$ by $g_{n}^{\prime}=\sum_{i \geq n} b_{i, n} f_{i}$ where the positive sequence $\left\langle b_{i, n}\right\rangle_{i \in \mathbb{N}}$ contains finitely many non null elements and $\sum_{i \geq n} b_{i, n}=1$. In fact, given that $\alpha^{*}$ is subadditive and positively homogeneous

$$
\alpha^{*}\left(g_{n}^{\prime}\right) \leq \sum_{i \geq n} b_{i, n} \alpha^{*}\left(f_{i}\right) \leq \sum_{i \geq n} b_{i, n} 2^{-i} \leq 2^{-n}
$$

Choosing weights conveniently we obtain, by Komlòs lemma [16, lemma A1.1, p. 515], that the sequence $\left\langle g_{n}^{\prime}\right\rangle_{n \in \mathbb{N}}$ converges $Q$ a.s. while by Egoroff theorem there is a set $F \in \mathcal{F}$ such that $Q\left(F^{c}\right)<\eta \epsilon$ and that, 
letting $g_{n}=g_{n}^{\prime} F$, the sequence $\left\langle g_{n}\right\rangle_{n \in \mathbb{N}}$ converges uniformly to some $g \geq 0$. As $0 \leq g_{n}^{\prime} \leq 1$

$$
Q(g)=\lim _{n} Q\left(g_{n}\right) \geq \lim _{n} Q\left(g_{n}^{\prime}\right)-Q\left(F^{c}\right) \geq \lim _{n} \eta\left(1-\epsilon_{n}-\epsilon\right)=\eta(1-\epsilon)
$$

while $\alpha^{*}(g)=\lim _{n} \alpha^{*}\left(g_{n}\right) \leq \lim _{n} \alpha^{*}\left(g_{n}^{\prime}\right)=0$. The implication $(b) \rightarrow(a)$ is obvious.

Acknowledgement 1. I owe my gratitude to Robert J. Elliot and William Sudderth for their kind encouragement and to M. Rasonyi for his comments. The usual disclaimer applies.

\section{REFERENCES}

[1] Agnew R. P., Morse A. P.: Extensions of Linear Functionals with Applications to Limits, Integrals, Measures and Densities, Annals of Mathematics, 39 (1938), 20-30.

[2] Aliprantis C., Border K.: Infinite Dimensional Analysis, Berlin - New York: Springer-Verlag 1998.

[3] Ansel J.P., Stricker C.: Lois de Martingale, Densités et Décompositions de Föllmer Schweizer, Annales de l'Institut Henri Poincaré 28 (1992), 375-392.

[4] Arrow K.: The Rôle of Securities in the Optimal Allocation of Risk Bearing, Review of Economic Studies, 31 (1964), 91-96.

[5] Back K.: Asset Pricing for General Processes, Journal of Mathematical Economics, 20 (1991), 371-395.

[6] Bättig R. J.: Completeness of Securities Market Models -An Operator Point of View, Annals of Applied Probability, 9 (1999), 529-566.

[7] Bättig R. J., Jarrow R. A.: The Second Fundamental Theorem of Asset Pricing: A New Approach, Review of Financial Studies, 12 (1999), 1219-1235.

[8] Black F., Scholes M.: The Pricing of Options and Corporate Liabilities, Journal of Political Economy, 81 (1973), $637-654$.

[9] Cassese G.: Some Finitely Additive Supermartingales, mimeo, 2005.

[10] Cassese G.: A Note on Asset Bubbles in Continuous Time, International Journal of Theoretical and Applied Finance, 8 (2005), 523-536.

[11] Clark S.: The Valuation Problem in Arbitrage Price Theory, Journal of Mathematical Economics 22 (1993), 463-478.

[12] Clark S.: Arbitrage Approximation Theory, Journal of Mathematical Economics, 33 (2000), 167-81

[13] Costantinides G., Duffie D.: Asset Pricing with Heterogeneous Consumers, Journal of Political Economy 104 (1996), 219-240.

[14] Daniel K. D., Hirshleifer D., Subrahmanyam A.: Investor Psychology and Security Market under- and Overreactions, Journal of Finance, $\mathbf{5 3}$ (1998), 1839-1885.

[15] Daniel K. D., Hirshleifer D., Subrahmanyam A.: Overconfidence, Arbitrage, and Equilibrium Asset Pricing, Journal of Finance, 56 (2001), 921-965.

[16] Delbaen F., Schachermayer W.: A General Version of the Fundamental Theorem of Asset Pricing, Mathematische Annalen 300 (1994), 463-520.

[17] Detemple J., Zapatero F.: Asset Prices in an Exchange Economy with Habit Formation, Econometrica, 59 (1991), 16331657.

[18] Dubins L.: Paths of Finitely Additive Brownian Motion Need not Be Bizarre, Séminaire de Probabilités XXXIII (1999), 395-396.

[19] Duffie D., Epstein L.: Asset Pricing with Stochastic Differential Utility, Review of Financial Studies, 5 (1992), $411-436$.

[20] Dunford N., Schwartz J. T.: Linear Operators. Part I, New York: Wiley 1988.

[21] Dybvig P., Ross. S.: Arbitrage, in Newman P. K., Milgate M., Eatwell J. (eds.) The New Palgrave Dictionary of Money and Finance, London: Macmillan 1992.

[22] Gilboa I., Schmeidler D.: Case-Based Decision Theory, Quarterly Journal of Economics, 110 (1995), 605-639.

[23] Glicksberg I. L.: A Further Generalization of the Kakutani Fixed Point Theorem, with Application to Nash Equilibrium Points, Proceedings of the American Mathematical Society, 3 (1952), 170-174.

[24] Harrison M., Kreps D. M.: Martingales and Arbitrage in Multiperiod Securities Markets, Journal of Economic Theory, 20 (1979), 381-408. 
[25] Harrison M., Pliska S.: Martingales and Stochastic Integrals in the Theory of Continuous Trading, Stochastic Processes and their Applications, 11 (1981), 215-260.

[26] Harrison M., Pliska S.: A Stochastic Calculus Model of Continuous Trading: Complete Markets, Stochastic Processes and their Applications, 15 (1983), 313-316.

[27] Jacod J., Shiryaev A.: Limit Theorems for Stochastic Processes, Berlin: Springer - Verlag 1987.

[28] Jarrow R. A., Rosenfeld E. R.: Jump Risks and the Intertemporal Capital Asset Pricing Model, Journal of Business, 57 (1984), 337-351.

[29] Kreps D. M.: Arbitrage and Equilibrium in Economies with Infinitely Many Commodities, Journal of Mathematical Economics, 8 (1981), 15-35. Annals of Applied Probability, 5 (1995), 906-925.

[30] Markowitz H. M., Perold A. F.: Portfolio Analysis with Factors and Scenarios, Journal of Finance, 36 (1981), $871-877$.

[31] Megginson R.: An Introduction to Banach Space Theory, Berlin: Springer - Verlag, 1998.

[32] Merton R. C.: An Intertemporal Capital Asset Pricing Model, Econometrica, 41 (1973), 867-887.

[33] Michael E.: Continuous Selections. I, Annals of Mathematics, 63 (1956), 361-382.

[34] Protter P.: Stochastic Integration and Differential Equations, Berlin: Springer - Verlag, 2004.

[35] Revusz D., Yor M.: Continuous Martingales and Brownian Motion, Springer-Verlag, Berlin 1999.

[36] Rubinstein M.: Implied Binomial Trees, Journal of Finance, 49 (1994), 771-818.

[37] Savage L. J.: The Foundations of Statistics, New York: Dover, 1972.

[38] Schmeidler D.: Subjective Probability and Expected Utility without Additivity, Econometrica, 57 (1989), $571-587$.

[39] Schweizer M.: Martingale Densities for General Asset Prices, Journal of Mathematical Economics, 21, 363-378 (1992).

[40] Stricker C.: Arbitrage et Lois de Martingale, Annales de l'Institut Henri Poincaré 26 (1990), $451-460$.

[41] Teigen, K.: Subjective Sampling Distributions and the Additivity of Estimates, Scandinavian Journal of Psychology, 24 (1974), 97-105.

[42] Tversky A.: Utility Theory and Additivity Analysis of Risky Choices, Journal of Experimental Psychology, 24 (1967), $27-36$.

[43] Tversky A., Koehler D.: Support Theory: A Nonextensional Representation of Subjective Probability, Psychological Review, 101 (1994), 547-567.

[44] Yosida K., Hewitt E.: Finitely Additive Measures, Transactions of the American Mathematical Society, 72 (1952), 46-66.

[45] Ziegler A.: Optimal Portfolio Choice under Heterogeneous Beliefs, European Finance Review, 4 (2000), 1-19.

University of Lugano and Università degli Studi di LeCCE

E-mail address: gianluca.cassese@lu.unisi.ch 